\title{
可见光促进的烯烃异构化反应
}

\author{
张 昊 $a, b$ 俞寿云 $*, b$ \\ $\left({ }^{a}\right.$ 扬州大学化学化工学院 扬州 225002) \\ ( $b$ 南京大学化学化工学院 生命分析化学国家重点实验室 南京 210023)
}

\begin{abstract}
摘要 传统合成热力学不稳定的 $Z$-式烯烃的方法往往都需要使用高能量的试剂, 通过动力学来进行控制, 反应条件较 为苛刻, 操作复杂, 原子经济性低下. 在分离的过程中, 也极易造成 $Z$-式烯烃向热力学更加稳定的 $E$-式烯烃的异构化. 随着有机光化学和光催化技术的不断发展, 越来越多的具有挑战性的有机合成反应可以通过有机光化学手段来解决. 总结了可见光促进的烯烃的异构化反应，并对其进行了展望.
\end{abstract}

关键词 光化学; 烯烃; 异构化; 能量转移

\section{Visible Light-Promoted Isomerization of Alkenes}

\author{
Zhang, Hao ${ }^{a, b} \quad \mathrm{Yu}$, Shouyun ${ }^{*, b}$ \\ ( ${ }^{a}$ School of Chemistry and Chemical Engineering, Yangzhou University, Yangzhou 225002) \\ ( ${ }^{b}$ State Key Laboratory of Analytical Chemistry for Life Science, School of Chemistry and Chemical Engineering, \\ Nanjing University, Nanjing 210023)
}

\begin{abstract}
The traditional methods for synthesizing $Z$-olefins generally require the use of high-energy reagents. These methods are usually controlled by kinetics. The reaction conditions are harsh and low atom economic. During the process of separation, Z-olefins are easy to convert to the thermodynamically more stable $E$-olefins. With the continuous development of organic photochemistry and photocatalysis technology, more and more synthetic challenges have been solved by photocatalysis. Herein, various types of visible light-promoted photocatalytic isomerization of olefins are reviewed.

Keywords photochemistry; olefins; isomerization; energy transfer
\end{abstract}

烯烃作为一种重要的结构单元, 广泛地存在于生物 活性分子和材料分子中. 在有机合成中, 烯烃也是最常 用的基团之一, 它们作为起始原料可以很容易地实现官 能团化, 来合成多种化学中间体和功能性骨架 ${ }^{[1]}$. 由于 烯烃的立体化学 $(Z / E$ 构型) 极大地影响着其化学、物理 和生理相关的性质及功能, 因此高度立体选择性地构建 烯烃一直都是有机合成重点的研究内容之一. 目前已经 有大量合成烯烃方法的报道, 比如, Wittig 反应及其改 良和变体 ${ }^{[2]}$ 、Julia 反应及其改良和变体 ${ }^{[3]}$ 、Peterson 烯 基化反应 ${ }^{[4]}$ 、烯烃复分解反应 ${ }^{[5]}$ 、炔烃的选择性还原 ${ }^{[6]}$ 等反应. 但这些方法往往得到的是烯烃立体异构体的混 合物. 通常来说, 选择性合成热力学稳定的 $E$-式烯烃的 方法已经比较成熟, 但是对于热力学不稳定的 $Z$-式烯烃 来说, 还是面临着很大的挑战. 目前合成 $Z$-构型烯烃的
反应往往依赖于反应的动力学控制, 通常都需要使用高 能量的试剂来促进 $Z$-式烯烃的合成 ${ }^{[7]}$. 然而在反应与分 离的过程中，也极易造成热力学不稳定的 $Z$-式烯烃向热 力学更加稳定的 $E$-式烯烃的异构化, 所以多数情况下 不能得到令人满意的立体选择性结果. 此外, 这些策略 所需要的反应条件也比较苛刻(如强碱和极低温度等), 并且同时还需要使用昂贵的过渡金属催化剂, 原子经济 性也比较低 ${ }^{[8]}$. 虽然烯烃的合成有了巨大的发展，但仍 然迫切地需要开发出简便高效绿色和立体选择性合成 烯烃的方法, 特别是 $Z$-式烯烃的合成.

近年来, 有机光化学和光催化技术, 特别是可见光 促进的化学反应, 得到了很大的发展, 越来越多的科学 家将其作为解决合成中长期存在的挑战的一种尝试. 本 文就近年来可见光促进的烯烃异构化的相关研究进展

\footnotetext{
* Corresponding author. E-mail: yushouyun@nju.edu.cn

Received September 6, 2018; revised October 14, 2018; published online October 19, 2018.

Dedicated to Professor Qingyun Chen on the occasion of his 90th birthday.

Project supported by the National Natural Science Foundation of China (No. 21732003).

国家自然科学基金(No. 21732003)资助项目.
} 
进行总结, 并且对相应的反应机理进行了讨论.

\section{1 烯烃的光化学异构化}

自然界中, 烯烃异构化最典型的例子就是在脊椎动 物光感受器中发生的 11-顺式视黄醛 1 异构化为全反式 视黄醛 2 的过程(Scheme 1). 该过程依赖于 11-顺式-视 黄醛 1 与视蛋白 3 以席夫碱的形式形成相应的视紫红质。 吸收可见光后导致了 11-顺式双键的几何异构化并形成 具有 11-反式双键的变视紫红质 II, 视紫红质构型的改 变, 启动了对大脑的神经脉冲, 从而产生视觉响应. 视 黄醛部分也会与视蛋白分离得到游离的 11-反式视黄醛 2 , 在光感受器中经过酶促还原后, 全反式视黄醇 $\mathbf{4}$ 转移 至视网膜色素上皮细胞中(RPE), 在卵磷脂视黄醇酰基 转移酶(LRAT)的作用下形成视黄酯 $\mathbf{5}$. 视黄酯 $\mathbf{5}$ 水解产 生的能量驱动了最后一步同分异构酶催化的 $E \rightarrow Z$ 的异 构化过程. 最后, 11-顺式视黄醇 6 被氧化成相应的 11顺式视黄醛 1 以完成视觉循环 ${ }^{[9]}$.

该过程表明可见光促进的烯烃的异构化是可行的. 受到此循环过程的启发, 同时在化学家们的不解努力之 下, 烯烃光敏化异构化的机理在理论和实验两个方面都 取得了较大的进展, 使得人们对光化学反应有了较为深 入的了解. 通常在基态反应中, $\mathrm{C}\left(\mathrm{sp}^{2}\right)-\mathrm{C}\left(\mathrm{sp}^{2}\right)$ 双键的旋转
往往受到高旋转能垒的阻碍，不易发生异构化. 普通的 烯烃也不能直接吸收可见光, 不能直接被可见光激发. 添加合适的光敏剂则可以克服这一困难 ${ }^{[10]}$. 可见光激 发光敏剂后，通过能量转移的方式敏化烯烃化合物，使 其跃迁至单线态，经系间审越过程得到双自由基的三线 态中间体，此中间体可以进行旋转来实现烯烃的异构 化. 由于不同构型的烯烃淬灭激发态光敏剂的速率是不 同的，通常共轭的 $E$-式烯烃可以有效地淬灭光敏剂，而 $Z$-式烯烃由于位阻作用, 是非共轭状态，通常不能有效 地淬灭光敏剂, 从而实现烯烃的 $E \rightarrow Z$ 异构化(Scheme $2)^{[11]}$. 高能量的紫外光直接照射也可以实现烯烃的激 发, 顺式烯烃激发所需要的能量要比反式烯烃激发所需 要的能量要高, 因此, 使用介于顺式与反式烯烃激发波 长之间的特定波长的光进行照射, 即可实现选择性地将 反式烯烃激发至三线态，经双自由基中间体的旋转后， 接着向顺式和反式两个方向发生衰减，而异构化得到的 顺式产物不会被再次活化, 所以其衰减系数的大小就决 定了最终的平衡体系中顺式产物与反式产物的比例，即 可选择性地得到烯烃定向异构化反应的顺式产物.

烯烃光化学异构化的基本策略主要有两种，一种是 在光敏剂的催化下，对既定构型的烯烃化合物直接实现 异构化; 另一种则是在通过化学转化构建双键的过程

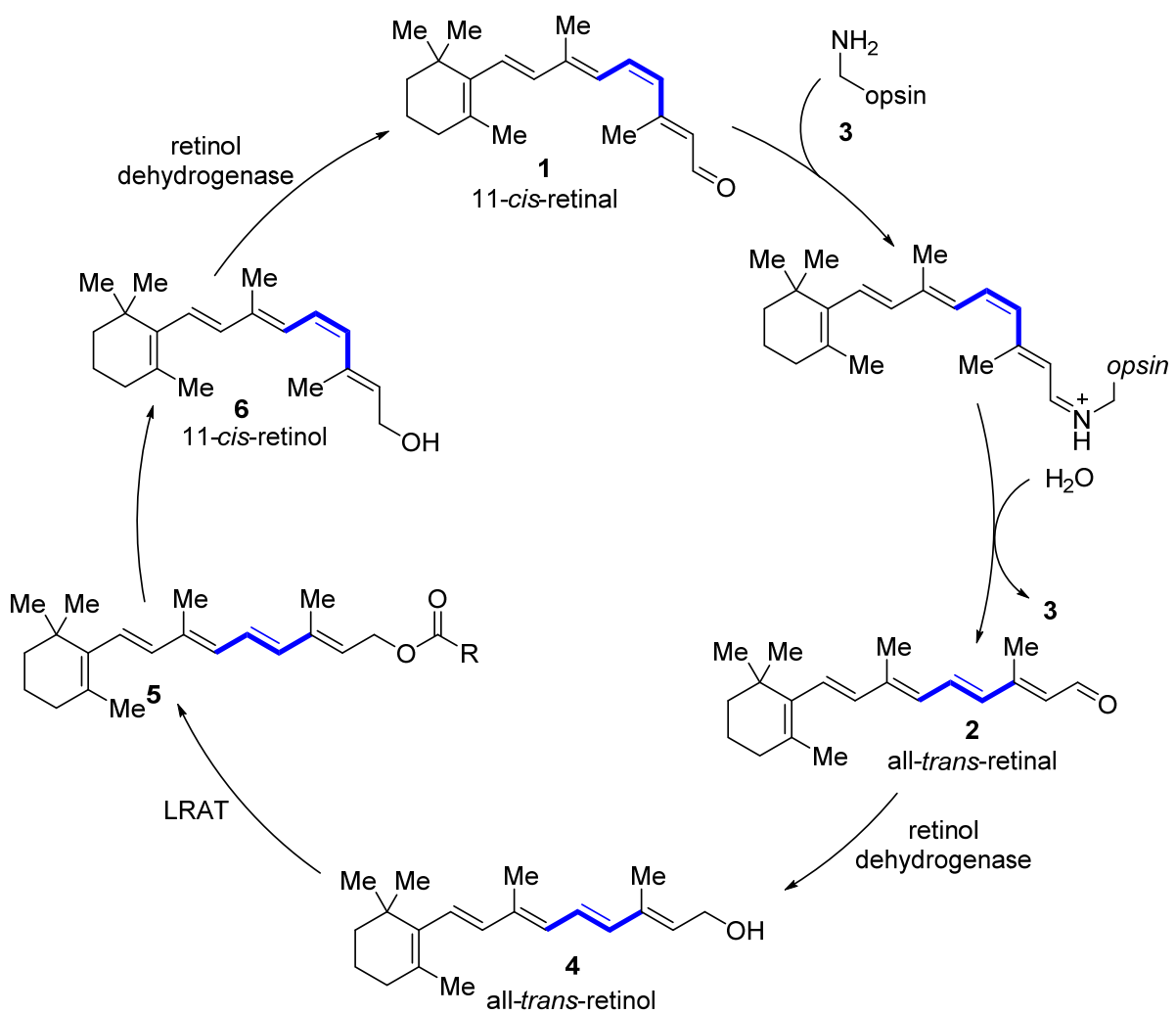

图式 1 视觉循环

Scheme 1 Visual cycle 


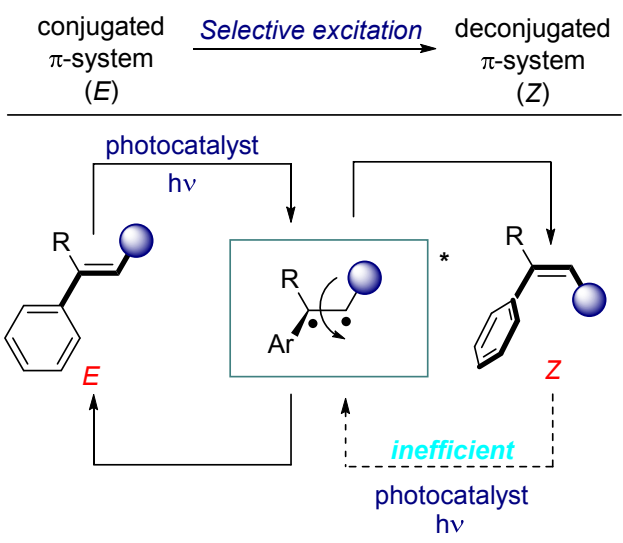

图式 2 光催化烯烃异构化的机理

Scheme 2 Conceptual framework for photocatalytic isomerization of alkenes

中, 对其进行立体选择性地控制.

\section{2 既定构型烯烃的光化学异构化}

\section{1 仿生的烯烃异构化}

1967 年, Walker 和 Radda ${ }^{[12]}$ 发现在紫外光的照射下, 全反式视黄醇 4 在(一)-核黄素[(-)-riboflavin]溶液中的 吸收显著降低，薄层色谱(TLC)显示有 11-顺式视黄醇 6 的生成(Scheme 3). 有趣的是, 在相同的条件下，使用 11-顺式视黄醇 6 同样可以得到逆向的异构化产物 4. 尽 管他们对核黄素催化的全反式视黄醇 4 反热力学的 $E \rightarrow Z$ 异构化过程只是局限于定性的研究, 但是这项研 究为烯烃选择性异构化指明了方向.

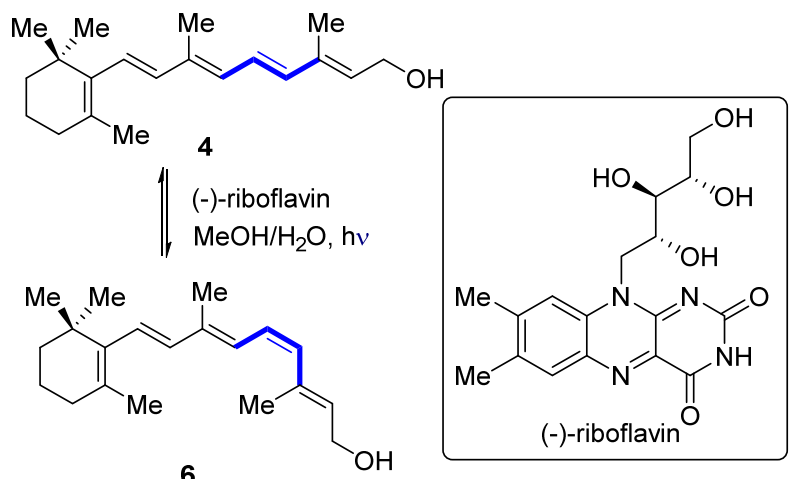

图式 3 核黄素介导的全反式视黄醇异构化为 11-顺式视黄醇 Scheme 3 ( - )-Riboflavin-mediated isomerization of all-transretinol to 11-cis-retinol

2015 年, Gilmour 等 ${ }^{[13]}$ 使用 Walker 所报道的廉价易 得的天然产物核黄素实现了活化烯烃的 $E \rightarrow Z$ 异构化 (Scheme 4). 该反应只需要添加 $5 \mathrm{~mol} \%$ 的(一)-核黄素, 而不需要其他任何添加剂, 在乙腈溶液中, 用 $402 \mathrm{~nm}$ 的 可见光照射 $24 \mathrm{~h}$, 即可实现转换, 反应操作简便, 条件
也比较温和. 研究表明, $\mathrm{R}^{2}$ 基团的引入与修饰可以显著 地提高异构化的选择性 $(\boldsymbol{Z}-7 \mathrm{c} \sim \boldsymbol{Z}-7 \mathrm{e})$. 但是 $\mathrm{F}$ 原子的引 入, 异构化效果并不是很理想, 可能是因为卤素的位阻 不足以使得顺式异构体的激发能垒增大很多. 对于芳环 底物来说, 无论是含有缺电子还是富电子基团, 其选择 性和反应收率都非常好 $(\boldsymbol{Z}-\mathbf{7 f} \sim \boldsymbol{Z}-\mathbf{7 h})$, 杂环化合物也同 样适用 $(\boldsymbol{Z}-\mathbf{7} \mathbf{i})$. 除了肉桂酸酯类的底物，他们进一步将 底物的适用性范围拓展到了醛 $(\boldsymbol{Z}-7 \mathbf{k})$ 、酮 $(\boldsymbol{Z}-7 \mathbf{l})$ 以及酰胺 $(\boldsymbol{Z}-7 \mathrm{~m})$ 等. 该反应也有其不足之处，反应底物受 $\mathrm{R}^{2}$ 基团 的影响非常大，同时烯烃也必须是缺电子的烯烃.

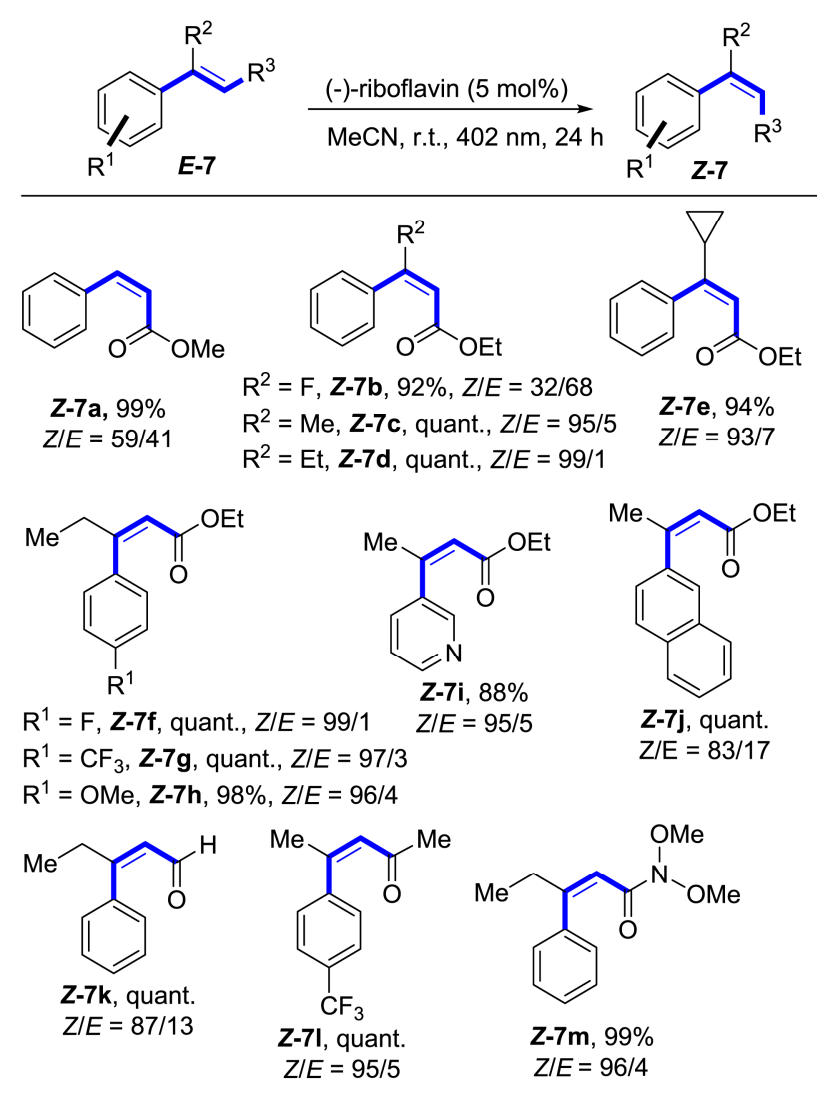

图式 4 核黄素催化的活化烯烃的异构化

Scheme 4 ( -)-Riboflavin-catalyzed isomerization of activated olefins

在上述异构化的最优条件下, Gilmour 等 ${ }^{[13]}$ 又对肉 桂酸衍生物 $\boldsymbol{E}-\mathbf{8}$ 进行了尝试. 有趣的是, 该底物成功地 以高选择性得到了预期的 $Z$-构型产物，但是产率却很 低，只有 $24 \%$ ，大部分反应产物却转化成了 4 -位取代的 香豆素 9. 当该异构化反应在氧气环境中进行时，分子 内环化产物 9 的收率可以高达 96\%. 这一发现为高效合 成香豆素这一重要的药物分子骨架提供了方法. 为了验 证上述反应广泛的适用性, Gilmour 等 ${ }^{[14]}$ 再次对一锅法 合成香豆素类化合物的反应条件进行了篮选和优化. 研 究表明, (一)-核黄素作为光催化剂, 体积比为 $1: 1$ 的 $\mathrm{MeCN} / \mathrm{MeOH}$ 的混合溶液是最佳的反应溶剂, 清洁高效 
的氧气是最理想的氧化剂, 在波长为 $402 \mathrm{~nm}$ 光的照射 下, 通过能量转移 $\left(E_{\mathrm{T}}\right)$ 实现反式肉桂酸双键的异构化, 随后经过单电子转移(SET)历程可以成功实现分子内环 化(Scheme 5). 考虑到(一)-核黄素经长时间光照会发生 降解, 于是他们每隔 $12 \mathrm{~h}$ 补加一次 $5 \mathrm{~mol} \%$ 的(一)-核黄 素以确保第二步的分子内环化反应不会受到前一步异 构化反应的影响. 此反应整体底物的适用性较好, 无 论芳环上是给电子或者是拉电子取代基, 产率都是 可以接受的.

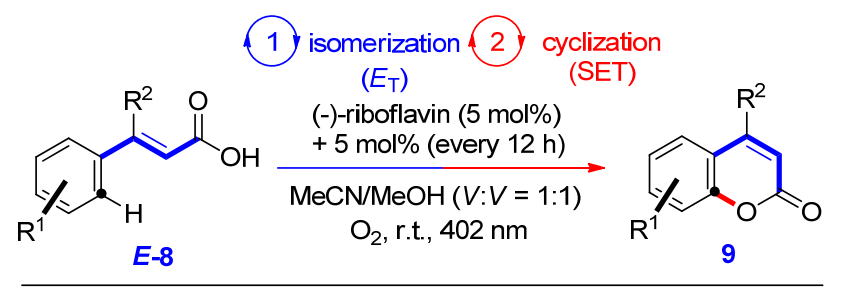

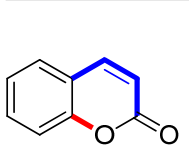

9a, $79 \%, 13.5 \mathrm{~h}$<smiles>Cc1cc(=O)oc2cc(Br)ccc12</smiles>

$9 e, 69 \%, 24 \mathrm{~h}$

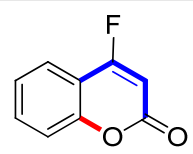

9b, $77 \%, 48 \mathrm{~h}$<smiles>Cc1cc(=O)oc2cc(C(F)(F)F)ccc12</smiles>

9f, $48 \%, 48 \mathrm{~h}$

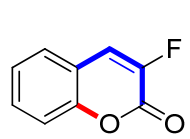

$9 c, 38 \%, 12 \mathrm{~h}$<smiles>Cc1cc(=O)oc2c1ccc1ccccc12</smiles>

$9 g, 52 \%, 8 \mathrm{~h}$

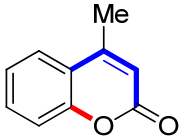

9d, $83 \%, 24 \mathrm{~h}$<smiles>[R]c1c([2H])c([2H])c([2H])c(/C(C)=C\C#N)c1[2H]</smiles>

Z-10m

quant., 91/9
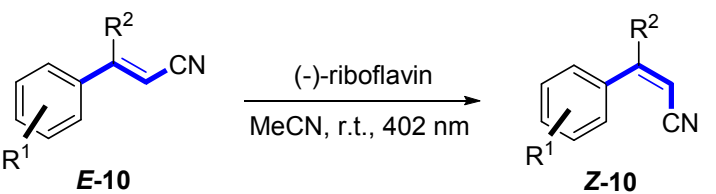

$\mathrm{R}^{2}=\mathrm{H}, \mathrm{Z}-10 \mathrm{a}$, quant., 63/37

$\mathrm{R}^{2}=\mathrm{Me}, \mathbf{Z}-10 \mathrm{~b}$, quant., 91/9 $\mathrm{R}^{1}=\mathrm{Cl}, \mathbf{Z}-10 \mathrm{e}$, quant., $88 / 2$

$\mathrm{R}^{2}=\mathrm{Et}, \mathbf{Z}-10 \mathrm{c}$, quant., $97 / 3 \quad \mathrm{R}^{1}=\mathrm{OMe}, \mathbf{Z}-10 \mathrm{f}$, quant., 81/19 $\mathrm{R}^{2}=$ cyPr, $\mathbf{Z}-\mathbf{1 0 d}, \mathbf{8 5} \%, 95 / 5 \mathrm{R}^{1}=\mathrm{CF}_{3}, \mathbf{Z}-\mathbf{1 0 g}$, quant., $91 / 9$<smiles>CC/C(=C/C#N)c1ccc(Br)cc1</smiles>

$\mathrm{R}^{1}=\mathrm{F}, \mathrm{Z}-10 \mathrm{~h}, 90 \%, 88 / 12$ $\mathrm{R}^{1}=\mathrm{OMe}, \mathrm{Z}-10 \mathrm{i}$, quant., $91 / 9$ $\mathrm{R}^{1}=\mathrm{CF}_{3}, \mathbf{Z - 1 0 j}$, quant., $97 / 3$
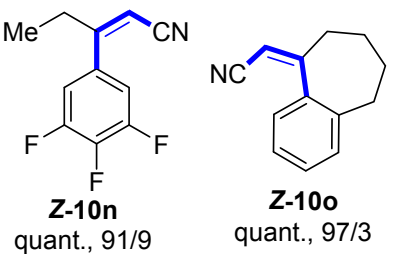

h, $61 \%, 6.5 \mathrm{~h}$
图式 5 光介导的核黄素催化合成香豆素

Scheme 5 Photocatalysis route to coumarins catalyzed by (一)-riboflavin

随后, Gilmour 等 ${ }^{[15]}$ 将同样的催化体系应用于肉桂 腈的光化学异构化中 (Scheme 6). 该研究中, 作者从底 物取代基的电子效应、位阻因素以及反应中温度和氧气 的影响等诸多方面进行了比较详尽的研究. 研究结果表 明, 在芳环上取代基不变的情况下, 在 $\beta$ 位引入取代基, 可以显著地提高 $Z$-异构体的选择性, 并且引入基团的位 阻越大, 选择性越好. 对芳环邻位取代基的研究, 也得 到了同样的结论, 这一结论与前期 $\alpha, \beta$-不饱和酯的光化 学异构化反应所观察到的现象一致. 值得一提的是, 通 过原子转移自由基聚合反应将(一)-核黄素负载于官能 才化的硅纳米粒子上, 并不会影响催化剂的光化学异构 化性能 ${ }^{[16]}$.

\section{2 羰基类化合物和稠环化合物作为光敏剂实现的烯 烃异构化}

羰基类化合物和多种稠环化合物作为三线态光敏 剂，可以用于烯烃的异构化反应，已经有很多报道 (Scheme 4). 早在 20 世纪 60 年代, Hammond 等 ${ }^{[17]}$ 就使 用苯并 $[a]$ 蒽 11 作为光催化剂, 实现了反式二苯乙烯的
图式 6 核黄素催化的肉桂腈的异构化

Scheme 6 ( - )-Riboflavin-catalyzed isomerization of cinnamonitriles

异构化 $\boldsymbol{E - 1 2}$ (Scheme 7). 随后他们又对二苯甲酮、苯乙 酮芴酮等一系列酮类化合物的三线态能量进行了测定， 并以 $450 \mathrm{~W}$ 的中压求灯作为光源，四甲基对苯醌 13 为 光敏剂, 反式 1,2-二苯基丙烯 $\boldsymbol{E}$-14 (1,2-Diphenylpropenes)作为研究对象, 用实验证实了顺反异构化反应确 实可以通过烯烃的三线态有效地发生 ${ }^{[18]} .20$ 世纪 80 年 代, Arai 等 ${ }^{[19]}$ 使用 2-䒺乙酮 15 作为光敏剂, 在 $1 \mathrm{~kW}$ 的 高压录灯的照射下，成功实现烷基取代的苯乙烯 $\boldsymbol{E - 1 6}$ 的 $E \rightarrow Z$ 异构化. 这些研究的共同特征是使用了化学计 量的光敏剂，尽管没有对最终产物进行分离，但是这也 已经很有力地证明了直接光照和光敏化都是很好的实 现富电子 $E$ 构型烯烃转化为相应的 $Z$ 构型烯烃的有效途 径.

2009 年, Liu 等 ${ }^{[20]}$ 借助三线态能量的理论, 尝试了 不同的酮类化合物作为光敏剂对多种烯烃进行光异构 化. 对不同骨架烯烃的尝试，将底物范围从二苯乙烯类 化合物扩展到共轭二烯、三烯等. 研究结果给出相同的 结论，烯烃骨架上的取代基位阻的增大，可以很大程度 上增大顺式烯烃的三线态能量，有助于反式烯烃向顺式 

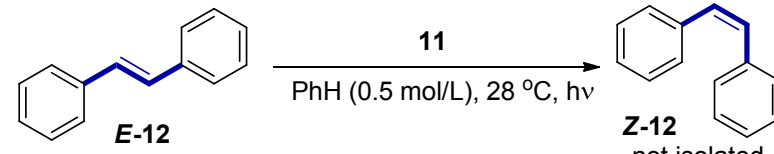

not isolated up to $92 / 8 \mathrm{Z} / E$

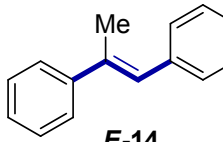

$E-14$

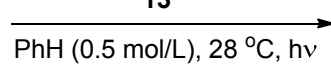<smiles>CC(C)(C)C(=O)/C=C/c1ccccc1</smiles>

$E-16$
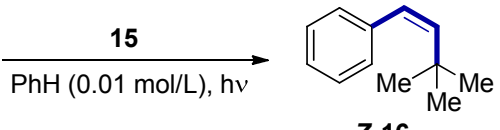

Z-16

not isolated up to $95 / 5 Z / E$

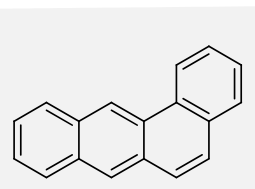

11

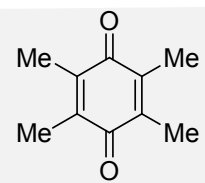

13

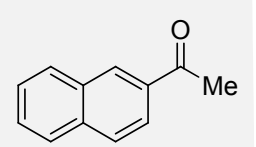

15
图式 7 稠环化合物或酮类化合物实现的 $E \rightarrow Z$ 异构化反应

Scheme $7 \quad E \rightarrow Z$ isomerization reaction used condensed nucleus compounds or ketones

烯烃的转换. 尽管此次报道的底物范围得到了一定拓 展, 但是所尝试的反应大多数需要使用紫外光作为光源 照射，顺式选择性也不理想，并没能发展成为具有应用 价值的通用方法.

2017 年, 王卫等 ${ }^{[21]}$ 对酮类光敏剂进行了进一步的 篮选和结构修饰，发展出了一种新型的酮类光敏剂 2碘-9-芴酮，并应用于可见光促进的烯烃异构化反应中， 实现了顺式二芳基乙烯类化合物的绿色高效合成. 其反 应机理如 Scheme 8 所示: 基态的 2-碘-9-芴酮作为光敏 剂, 在可见光的驱动下, 被激发至第一激发单线态, 接 着发生系间窝越变成激发的三线态, 三线态中间体随后 与基态的反式烯烃发生能量交换, 使得双键断裂形成双 自由的中间体, 经过 $\mathrm{C}-\mathrm{C}$ 键的旋转, 最后得到相应的 顺式烯烃. 通过对二苯乙烯类衍生物在该反应条件下底 物适用性的研究发现, 对于对位取代的二苯乙烯类底 物，无论是吸电子基团或者是给电子基团，都可以得到 高收率并且顺反选择性较好的产物 $(Z-17 a 、 Z-17 b)$. 而 当取代基在邻位时, 反应收率与选择性都有显著的提升 $(\boldsymbol{Z}-17 \mathrm{e} \sim \boldsymbol{Z}-17 \mathrm{e})$, 这很可能是因为邻位取代基的引入, 使得顺式构型产物的结构在空间上变得更加拥挤, 进而 增大了顺势产物三线态的能量, 而对于反式底物来说, 空间上的结构仍然相对比较平坦, 三线态的能量变化并 不大，最终导致了异构化的发生.

2017 年, Gilmour 等 ${ }^{[22]}$ 报道了光诱导的葱催化的烯

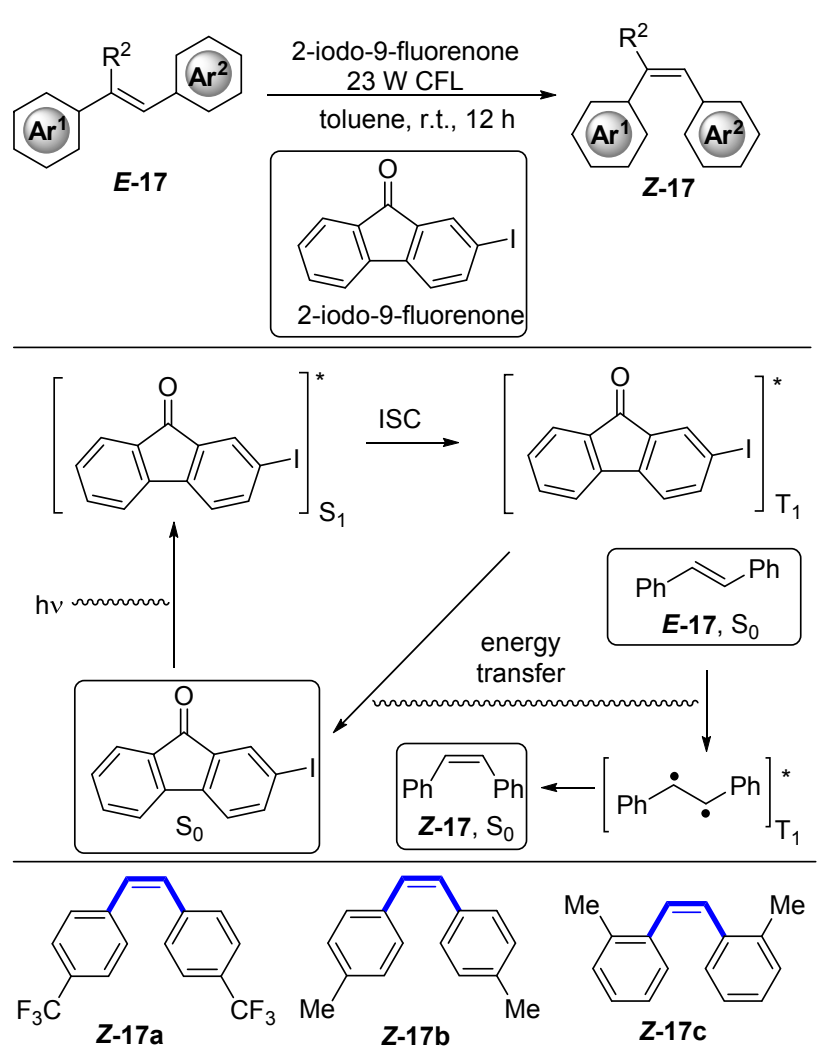

$93 \%, Z / E=83 / 17 \quad 95 \%, Z / E=82 / 18$

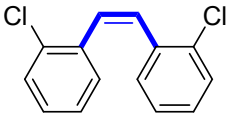

Z-17d

$99 \%, Z / E=97 / 3$

$\mathrm{Me}$

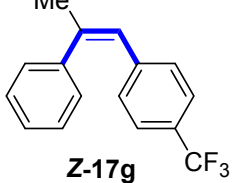

$93 \%, Z / E=92 / 8$

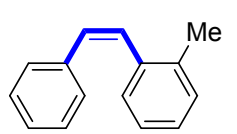

Z-17e $99 \%, Z I E=92 / 8$

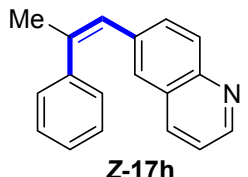

$95 \%, Z / E=75 / 25$
$99 \%, Z / E=99 / 1$

$\mathrm{Me}$

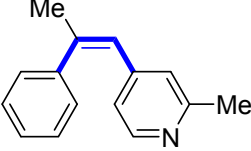

Z-17f

$95 \%, Z / E=97 / 3$

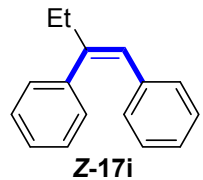

$99 \%, Z / E=99 / 1$
图式 8 光催化的二苯乙烯类衍生物 $E \rightarrow Z$ 异构化 Scheme 8 Photocatalytic $E \rightarrow Z$ isomerization of stilbene derivatives

丙醇 18 的 $Z \rightarrow E$ 异构化反应，并通过钯催化的氧化环化 反应构建 4-三氟甲基取代的 $2 H$-色烯类化合物 19 (Scheme 9). 研究发现, 当使用 $Z$-构型的烯丙醇作为起 始原料时，即使升高温度也不能使得环化反应发生，而 通过光催化的方式, 即可成功地将 $Z$-式的烯丙醇异构化 为 $E$-式产物, 在醋酸钯的催化下, 通过环化得到相应的 三氟甲基取代的 $2 H$-色烯类化合物.

\section{3 金属配合物作为光敏剂实现的烯烃异构化}

多联吡啶配合物如 $\mathrm{Ru}(\mathrm{II}), \mathrm{Ir}(\mathrm{III})$ 等, 基于其电子到 配体的电子转移(metal-to-ligand charge transfer, MLCT) 激发态具有优良的光化学与光物理性质，而被广泛地用 作可见光激发的光敏剂. 其光敏作用主要是通过分子内 

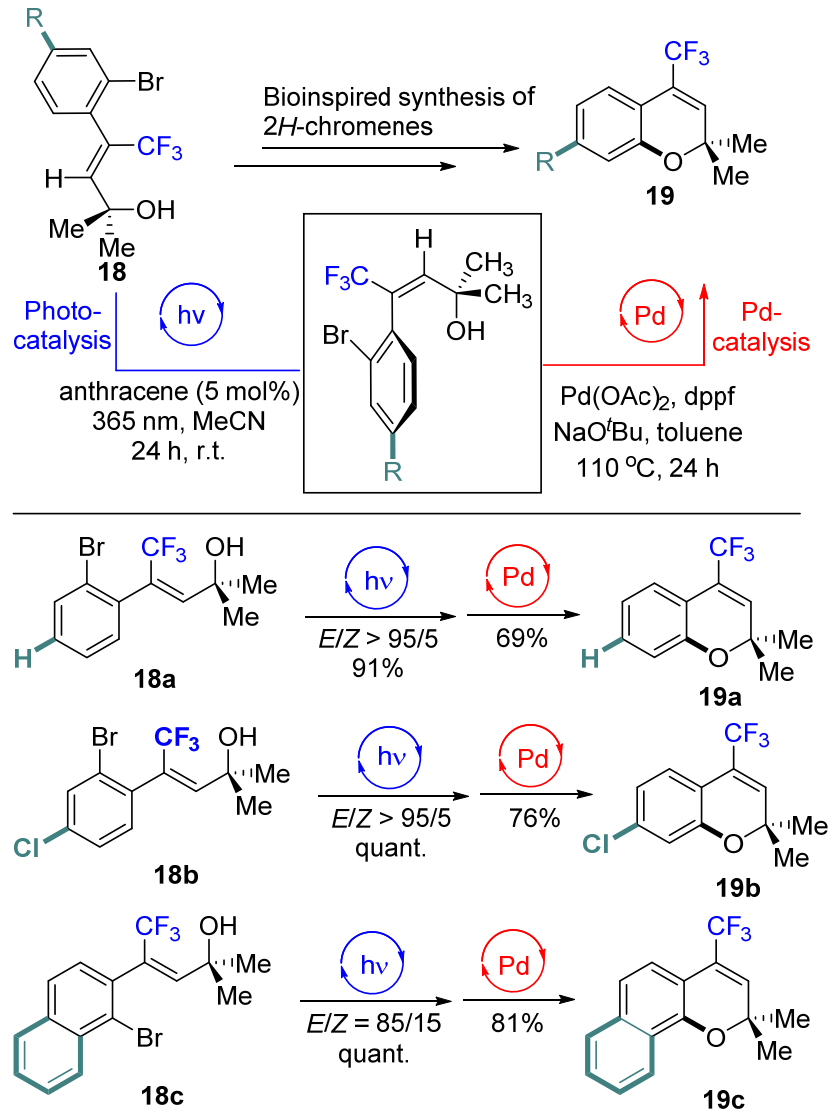

图式 $92 H$-色烯类化合物的简洁合成

Scheme 9 Concise synthesis of $2 H$-chromenes

的能量转移及(或)电子转移来对底物进行活化.

Osawa 等 ${ }^{[23]}$ 在 2001 年报道了在多联吡啶 Ru(II) 配合 物上通过共价键引入叔膦配体来实现反式-4-氰基-二苯 乙烯 $(\boldsymbol{E}-\mathbf{2 0})$ 的光异构化反应(Scheme 10). 此反应使用 2 $\mathrm{mol} \%$ 的含膦多联吡啶 Ru(II)配合物作为光催化剂, 在可 见光的照射下 (400 W lamp, $\lambda>450 \mathrm{~nm})$, 室温条件下反 应 $2 \mathrm{~h}$ 即可得到顺式二苯乙烯 $\boldsymbol{Z - 2 0}$ 为主的混合物 $(Z / E=$ $85 / 15)$, 尽管他们当时没有尝试更多的底物，但是这个 方法的提出为使用金属配合物作为光敏剂, 实现可见光 诱导的烯烃异构化反应提供了一个新的思路.

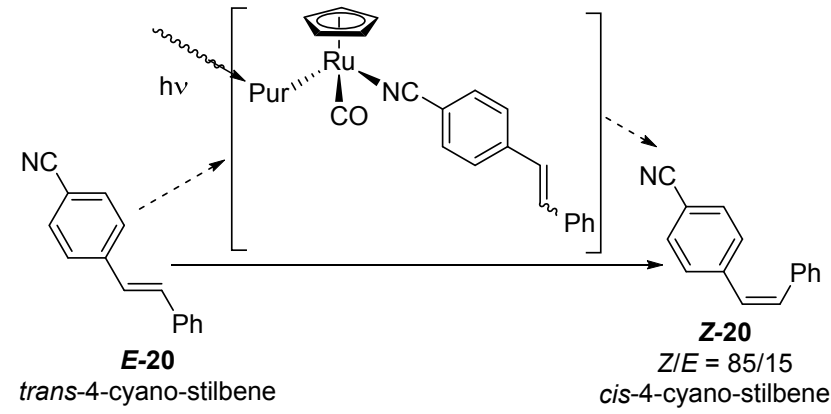

图式 10 可见光诱导的 4-氧基二苯乙烯的 $E \rightarrow Z$ 异构化 Scheme 10 Photocatalytic $E$-to- $Z$ isomerization of 4-cyanostilbene
2014 年, Weaver 等 ${ }^{[7]}$ 报道了铱配合物作为光催化剂 介导的反式芳基烯丙基胺的异构化反应. 此反应使用 $0.7 \mathrm{~mol} \%$ 的 $f a c-\operatorname{Ir}(\mathrm{ppy})_{3}$ 作为光催化剂, 在蓝色 LED 灯的 照射下，可以以最高 $96 \%$ 的收率得到反式芳基烯丙基胺 $\boldsymbol{E - 2 1}$ 的异构化产物 Z-21, 分离前的选择性高达 98/2. 该 反应底物整体官能团的容忍性较好，原子经济性高，有 比较好的应用前景. Weaver 就异构化反应提出了两种可 能的 $\operatorname{Ir}(\mathrm{ppy})_{3}$ *催化剂光淬灭的机理, 如 Scheme 11 所示. 第一种是还原淬灭机理: 底物胺 $\boldsymbol{E}-22$ 通过单电子转移 过程( single electron transfer, SET)过程被激发态的 $\operatorname{Ir}(\mathrm{III})$ *氧化成氮正离子自由基 $\boldsymbol{E}-22 \mathrm{a}$. 随着胺被氧化, 使得氮原子 $\alpha$-位 $\mathrm{H}$ 的酸性得到增强，可以被外加碱可逆 性地去质子化, 得到烯丙基自由基 $\boldsymbol{E}-\mathbf{2 2 b}$. 烯丙基自由 基 $\boldsymbol{E}-22 \mathrm{~b}$ 可以发生双键的异构得到 $\boldsymbol{Z}-22 \mathrm{~b}, \boldsymbol{Z}-22 \mathrm{~b}$ 进一步 地进行质子化变成 $Z-22 \mathrm{a}$, 最终经历反向的单电子转移 被还原成最终顺式产物 $Z$-22. 另一种则是双自由基的 机理: 激发态的光敏剂通过能量转移(energy transfer,

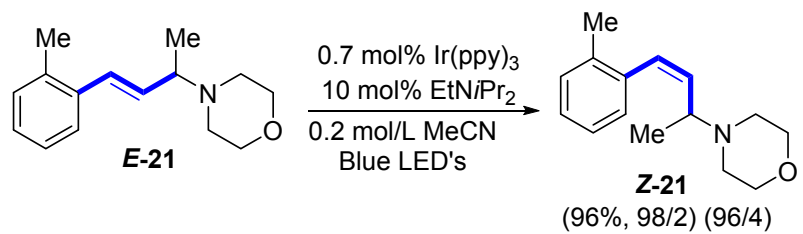

(isolated yield, isolated $Z / E$ ) (crude $Z / E$ )
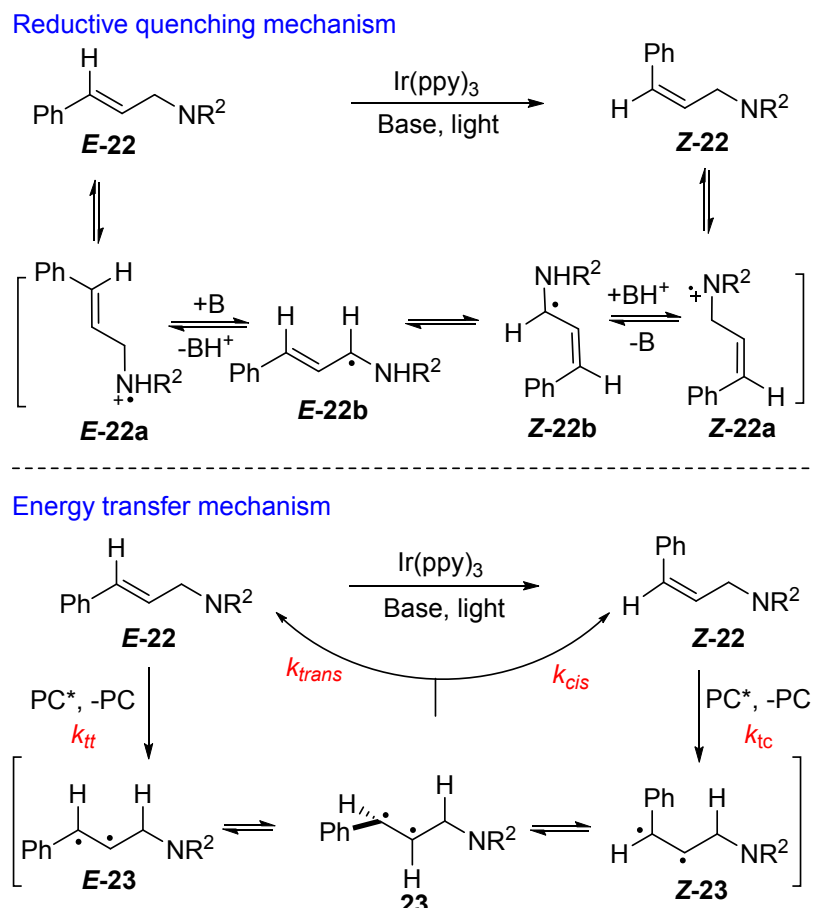

图式 11 两种可能的可见光催化的烯丙基胺的 $E \rightarrow Z$ 异构化 的机理

Scheme 11 Two possible mechanism of the photocatalytic $E$-to- $Z$ isomerization of allylic amine 
$E_{\mathrm{T}}$ )过程将能量传递给底物 $\boldsymbol{E - 2 2}$, 生成双自由基中间体 23, 该中间体可以围绕 $\mathrm{C}-\mathrm{C}$ 键进行 $90{ }^{\circ} \mathrm{C}$ 的旋转得到 具有临界立体构型的中间体, 由于第一步的速率常数更 大, 所以进一步通过系间窝越(Intersystem Crossing, ISC)得到以顺式构型为主的产物 $\boldsymbol{Z - 2 2}$.

除了对胺类取代的烯烃进行异构化研究外, Weaver 等 ${ }^{[7]}$ 还对其他类型取代的烯烃, 包括烃类 $(\boldsymbol{E}-\mathbf{2 4 a}, \boldsymbol{E}-$ $25 a) 、$ 醇类 $(\boldsymbol{E}-26 \mathbf{a})$ 和酯类 $(\boldsymbol{E}-27 \mathbf{a})$ 等进行了尝试, 均可得 到有用的选择性与收率. 这也证明了从热力学稳定的 $E$ 构型烯烃出发, 通过光催化直接获得热力学不稳定的 $Z$ 构型烯烃的可行性, 底物的范围也可以进一步地拓宽.

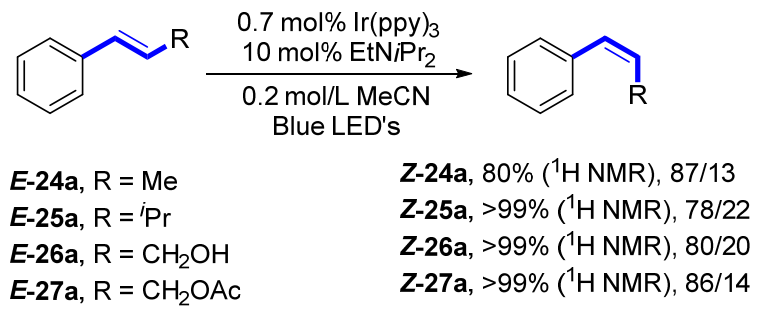

Reiser 等 ${ }^{[24]}$ 使用 $f a c-\operatorname{Ir}(\mathrm{ppy})_{3}$ 作为光催化剂, 同样实 现了化合物 $\boldsymbol{E - 2 8}$ 的 $E \rightarrow Z$ 异构化反应(Scheme 12). 他们 推断此反应仍然是通过双自由基中间体的转换而来. 有 趣的是, 他们发现此异构化反应在较高的温度 $\left(90{ }^{\circ} \mathrm{C}\right)$ 中进行并不会导致最终的 $Z / E$ 比例发生改变.

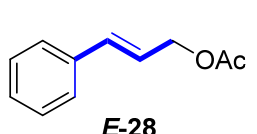

$E-28$

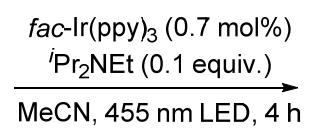

via:<smiles>CC(=O)OCC=Cc1ccccc1</smiles>

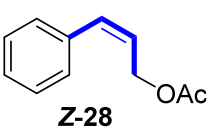

ZIE $=82 / 18$

图式 12 可见光诱导的化合物 $\mathbf{2 8}$ 的 $E \rightarrow Z$ 异构化 Scheme 12 Photocatalytic $E$-to- $Z$ isomerization of compound 28

2015 年, Rueping 等 ${ }^{[25]}$ 报道了一种反应条件温和的, 可见光诱导的二苯乙烯类化合物 $\boldsymbol{E - 2 9} E \rightarrow Z$ 异构化反应 (Scheme 13). 该反应使用 $3 \mathrm{~mol} \%$ 的铱配合物作为光催 化剂, 通过 $11 \mathrm{~W}$ 苂光灯照射, 在氧气的氛围下实现完 全的构型反转, $Z / E$ 选择性高达 $99 / 1$, 而反应的收率也接 近定量. 此反应最大的优势在于其操作的简便性与异构 化效率的高效性. 此外, Rueping 等 ${ }^{[25]}$ 还极具创造性地 将此异构化反应应用于有机一离子液体两相溶液的非均 相体系. 光催化剂由于所带电荷而溶于离子液体中, 反 应的底物则溶于有机相中, 反应完成后, 有机相可以直 接进行分离, 而离子液体相则可以继续循环使用, 实验 结果表明, 在循环使用 8 次后, 此反应体系仍然可以保 证 $89 \%$ 的收率. 最后他们又将此异构化过程设计成一种

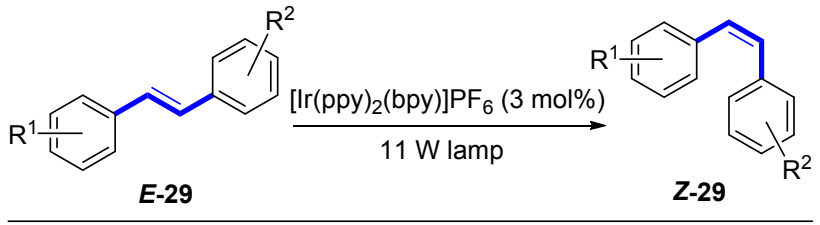

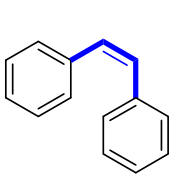

Z-29a, 98\% $Z / E=99 / 1$<smiles>Cc1ccc(/C=C\c2ccccc2)cc1</smiles>

Z-29d, 93\% $Z / E=12 / 1$<smiles>COc1cccc(/C=C\c2ccccc2OC)c1</smiles>

Z-29b, 98\% $Z / E=99 / 1$<smiles>Clc1ccccc1/C=C\c1ccccc1</smiles>

Z-29e, 98\% $Z / E=99 / 1$<smiles>Clc1ccc(/C=C\c2ccc(Cl)cc2)cc1</smiles>

Z-29c, 96\% $Z / E=99 / 1$

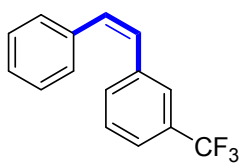

Z-29f, 98\% $Z / E=99 / 1$

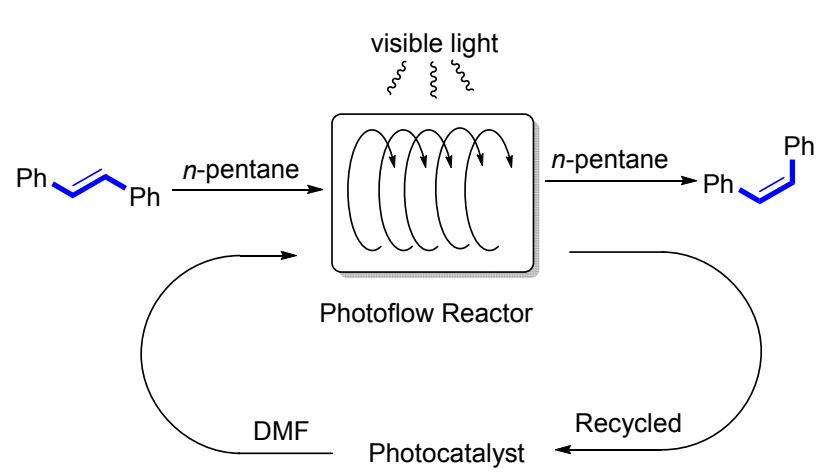

图式 13 催化的二苯乙烯类化合物 $E \rightarrow Z$ 异构化

Scheme 13 Photocatalytic $E$-to- $Z$ isomerization of diverse stilbene derivatives

连续流动化学的反应. 该方法的建立可以使得流速控制 在 $10 \sim 20 \mathrm{~mL} / \mathrm{h}$ 的底物在大功率的蓝灯照射下, 实现定 量的异构化反应，并且可以放大到克级规模.

2018 年, Gilmour 等 ${ }^{[26]}$ 在前期对极化烯烃异构化工 作的理论基础上, 发展了可见光诱导的烯基硼酸酯反热 力学的 $E \rightarrow Z$ 异构化反应(Scheme 14). 该反应是通过能 量转移过程实现的. 共轭的 $\pi$ 系统被激发态的铱配合物 活化, 得到的瞬时双自由基的中间体围绕着 $\mathrm{C}-\mathrm{C}$ 键进 行旋转, 产生顺式和反式两种异构体. 其中, 顺式异构 体中烯丙位的张力使得 $\pi$ 系统产生去共轭效应，而不能 再次被活化. 促使整个平衡趋向于顺式产物的生成. 该 反应条件温和，操作简便，芳环上吸电子基团与供电子 基团都可以容忍，尤其是对 $\beta$-位具有取代基的烯基嗍酸 酯也可以很好的调控.

为了进一步凸显此异构化在合成中的应用价值, Gilmour 等 ${ }^{[26]}$ 进行了相应的硼酸酯的衍生化. 使用 $Z-30 a$ 作为起始原料, 顺利地与 4-溴苯甲酸乙酯 31 偶联 

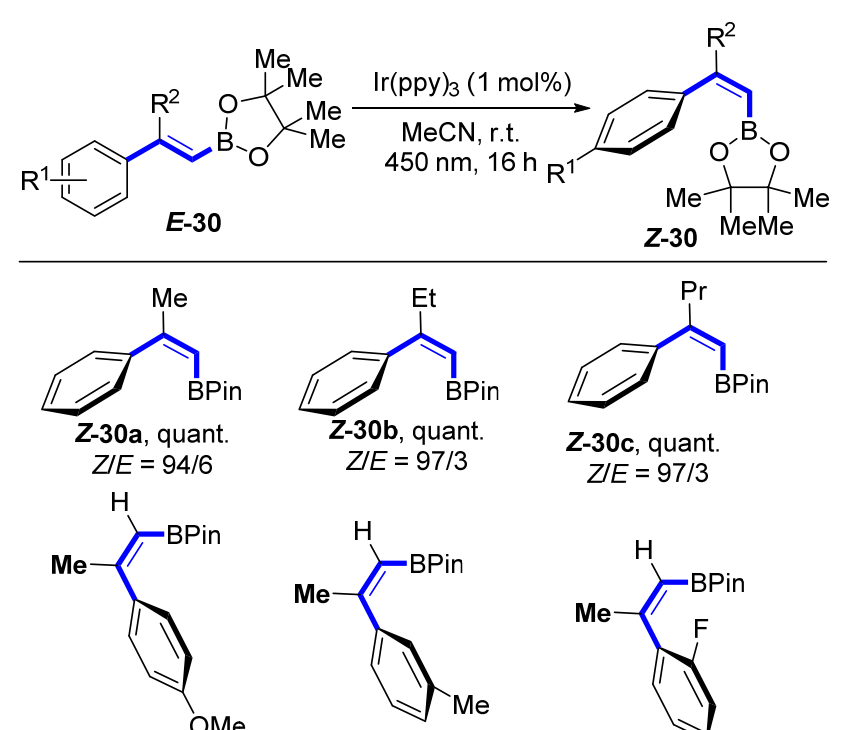

$\mathrm{OMe}$
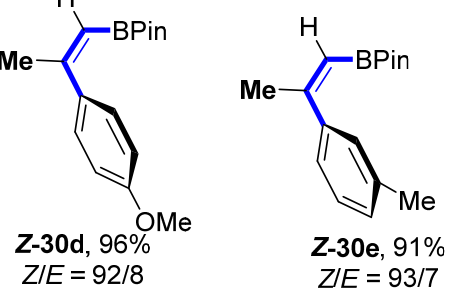

ZIE $=97 / 3$

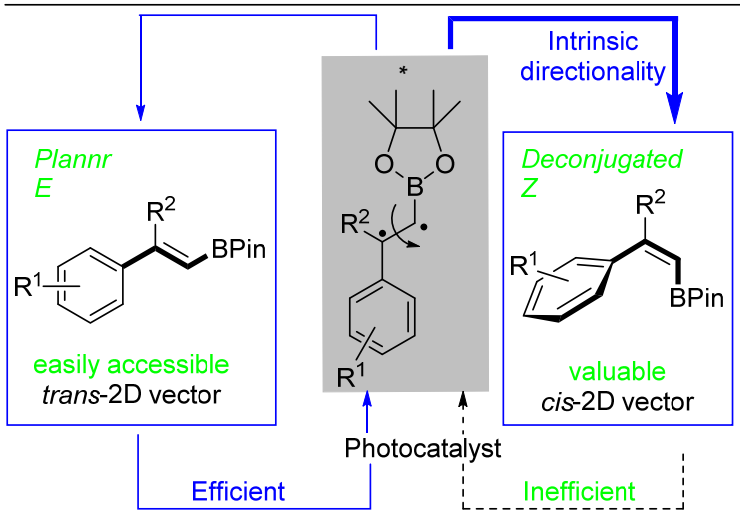

图式 14 光催化的反式- $\alpha$-取代的烯基嗍酸酯的 $E \rightarrow Z$ 异构化 Scheme 14 Photocatalytic $E \rightarrow Z$ isomerization of trans- $\alpha$ substituted styrenyl BPins

得到 Suzuki-Miyaura 反应的产物 32; 在氧化偶联的条件 下，与丙烯酸甲酯 33 生成烯基化产物 34. 碘的取代反 应以及氟嗍酸钾物种 36 的制备都可以顺利地进行 (Scheme 15).

\section{3 立体选择性控制烯烃的合成}

2014 年, 卿风翎等 ${ }^{[27]}$ 在此前使用过渡金属介导和 催化的三氟甲基化反应的研究基础上, 开发了一种新型 的可见光诱导的苯乙烯的直接三氟甲基化方法, 更重要 的是, 此方法可以通过选择特定的光催化剂来实现三氟 甲基化后烯烃的 $E / Z$ 构型的控制(Scheme 16). 热力学稳 定的 $E$-异构体可以通过钓配合物催化的 SET 循环来直 接构建, 而热力学不稳定的 $Z$-异构体则是依赖于铱配合 物催化的 SET/TTET “一锅法” 的过程来制备. 这是第 一例可调节的立体选择性合成三氟甲基化烯烃的方法. 研究表明, 在苯乙烯芳环上引入给电子基团, 使得氧
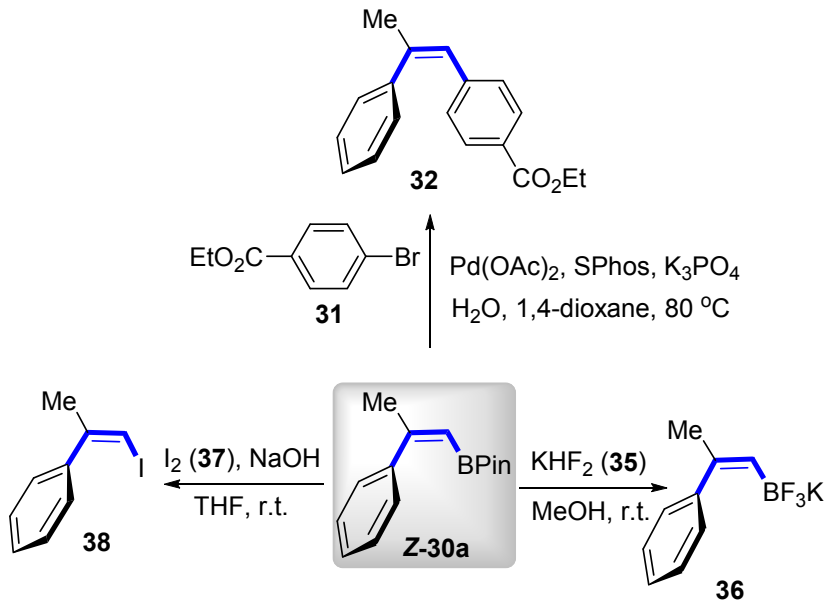

$\mathrm{Pd}(\mathrm{OAc})_{2}, \mathrm{O}_{2}$
$\mathrm{DMA}, 50^{\circ} \mathrm{C}$$\prod_{\mathrm{O}}^{\mathrm{O}} \mathrm{CH}_{3}$

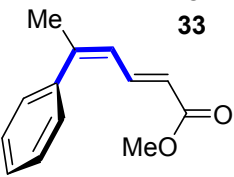

34

图式 15 嗍酸酯的转化

Scheme 15 Conversions of styrenyl BPins

化淬灭循环中碳自由基更容易被氧化成碳正离子中间 体. 此外, 给电子基团处于苯乙烯的邻位，也可以有效 地防止碳正离子中间体受到分子间的亲核进攻. 这两点 可以有效地避免副产物的生成，以提高反应收率.

同年, Cho等 ${ }^{[28]}$ 报道了光催化的炔烃的双官能团化 反应(Scheme 17). 研究发现, 反应条件中催化剂、碱和 溶剂的差别, 决定了产物 $Z / E$ 的选择性. 当使用 $\left[\mathrm{Ru}(\mathrm{phen})_{3}\right]-\mathrm{Cl}_{2}$ 作为光催化剂时, 炔烃 $\mathbf{4 1}$ 与三氟硒甲烷 42主要得到的是炔烃双官能团化的 $E$-产物 43 , 选择性可 以高达 $18: 1$, 收率也较高. 当使用 $f a c-\operatorname{Ir}(\mathrm{ppy})_{3}$ 作光催化 剂, 1,8-二氮杂双环 [5.4.0]十一碳-7-烯(DBU)作碱, 在乙 腈/四氢呋喃的混合溶剂中反应时，则发生炔烃的氢三 氟甲基化反应，产物 44 以 $Z$-式为主，但是选择性有待提 高.

Weaver 等 ${ }^{[29]}$ 从 2014 年开始就对光诱导的 $C-F$ 键 活化进行了一系列的研究. 在前期成功实现多氟芳烃烷 基化和芳基化 ${ }^{[30]}$ 的基础上，他们使用相同的策略，在可 见光的激发下，使用多氟芳烃自由基对取代炔烃 46 加 成得到骨架多样的烯基化多氟芳烃化合物，并且通过对 光催化剂的篎选来实现对烯基化产物立体选择性的控 制 ${ }^{[31]}$. 该反应也是电子转移与能量转移在同一个反应 中共同发生的鲜有报道例子之一(Scheme 18). 光催化 剂在可见光的照射下, 吸收一个光子后变成激发态, 经 过还原淬灭的过程产生中间体，接着发生单电子转移， 


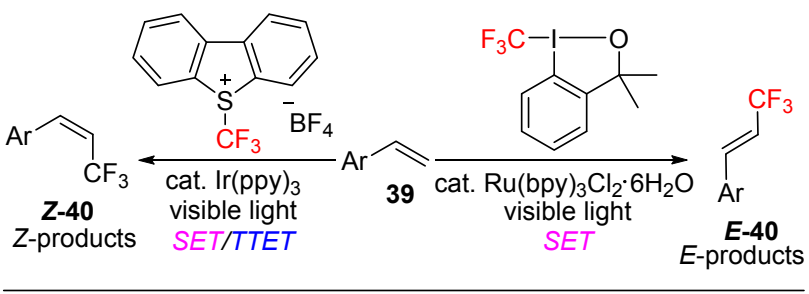

E-Trifluoromethylatedalkenes:

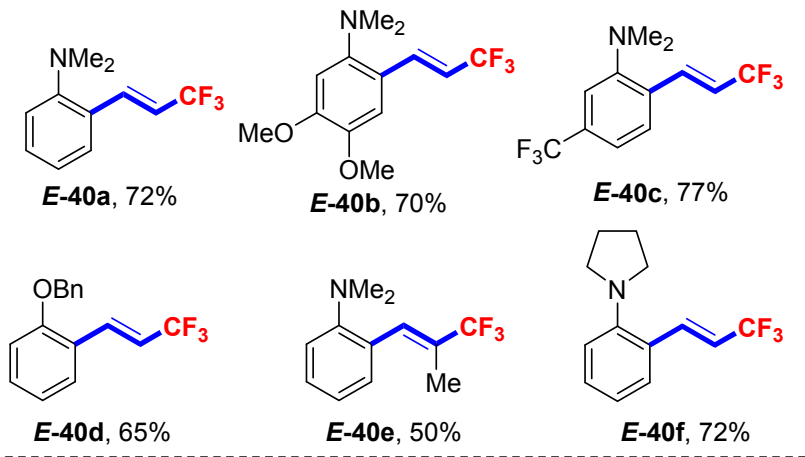

Z-Trifluoromethylated alkenes:

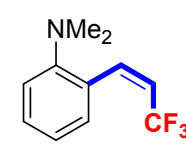

Z-40a, $65 \%$ $Z I E=9.3 / 1$

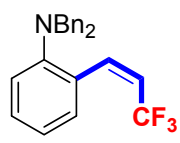

Z-40d, 75\% $Z I E=5.2 / 1$
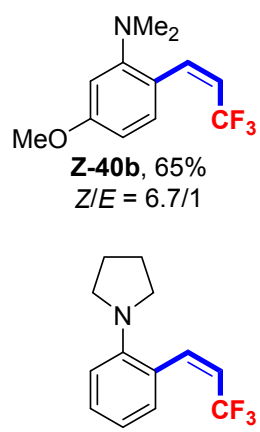

Z-40e, $55 \%$ $Z / E=10 / 1$
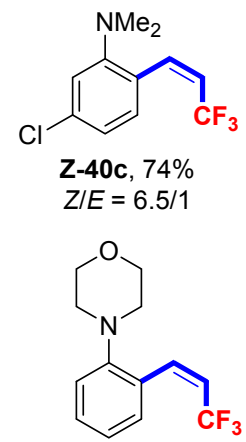

Z-40f, $86 \%$ $Z / E=7.1 / 1$
图式 16 苯乙烯类化合物选择性的三氟甲基化

Scheme 16 Stereoselective trifluoromethylation of styrenes

多氟芳烃 45 得到一个电子后脱去一个氟负离子以产生 多氟芳烃自由基中间体，该中间体会进一步对炔烃进行 自由基加成得到乙烯基自由基, 乙烯基自由基再经过氢 原子转移(HAT)过程㩲取氢原子形成苯乙烯类似物, 其 顺反的选择性取决于炔烃上 $\mathrm{R}$ 基团的大小. 最后，苯乙 烯类似物与激发态的光催化发生能量交换, 有趣的是, 研究表明能量转移的速率与光催化剂体积的大小有着 密切的关系, 大体积的光催化剂能量转移的速率较慢, 所以异构化的平衡最终趋向于反式构型，反之亦然.

2016 年, 黄学良等 ${ }^{[32]}$ 报道了可见光诱导的钯/钉协 同催化芳基碘化物 48 和烯烃的 Heck 偶联反应(Scheme 19). 该反应无需添加配体，条件温和，室温下即可进 行, 同时也避免了使用紫外光等高能量光源. 通过大量 的条件篮选以及控制实验的验证，他们发现使用 $\mathrm{Ru}(\mathrm{bpy})_{3} \mathrm{Cl}_{2} \cdot 6 \mathrm{H}_{2} \mathrm{O}$ 与 $\mathrm{Pd}(\mathrm{OAc})_{2}$ 协同催化效果最为理想, 可以以最高 $99 \%$ 的收率得到相应的 $E$-肉桂酸酯 $\mathbf{5 0}$. 研
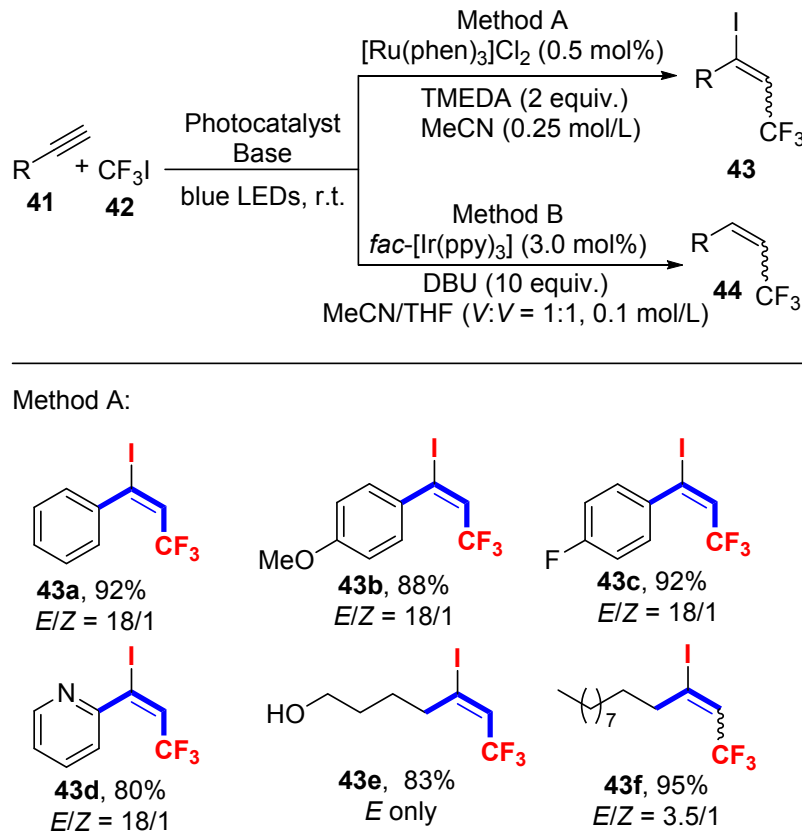

Method B:

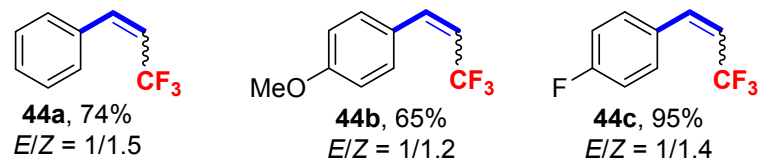

图式 17 可见光催化的炔烃的三氟甲基化反应

Scheme 17 Trifluoromethylation reactions of alkynes through visible light photoredox catalysis

究表明，碘化物芳环上的电性不会对反应性造成影响 (50a 50c). 芳环上存在未保护的羟基或氨基的底物同 样可以顺利地反应 $(50 \mathrm{~d} \sim 50 \mathrm{e})$ ，虽然收率有所降低，但 是反应整体底物范围广泛，对官能团的容忍度较好，取 代基的位阻也不会影响产率与立体选择性. 值得注意的 是，当使用苯乙烯类化合物 $\mathbf{5 1}$ 与芳基碘化物 $\mathbf{4 8}$ 偶联时， 产物会出现 $E \rightarrow Z$ 异构化现象, 通过增加 $\mathrm{Ru}(\mathrm{bpy})_{3} \mathrm{Cl}_{2}$ ・ $6 \mathrm{H}_{2} \mathrm{O}$ 与 $\mathrm{Pd}(\mathrm{OAc})_{2}$, 可以成功地控制产物的立体构型, 最终得到高选择性的 $Z$-二芳基乙烯 $\mathbf{5 2}$.

王否等 ${ }^{[33]}$ 报道了一种可见光控制的三组分参与的 合成菲啶类化合物的自由基串联反应(Scheme 20)。该 反应是通过芳基亚磺酸 53 与联芳基异氰 55 形成电子给 体受体(EDA)复合物后被光化学激发所驱动. 研究发现, 室温下使用 $3 \mathrm{~W}$ 的蓝灯照射可以得到热力学稳定的 $E$ 式菲啶类化合物, 而使用 $3 \mathrm{~W}$ 的紫外灯进行照射, 产物 的构型则以 $Z$-式为主. 该串联反应显示出了很高的区域 和立体选择性，反应条件温和，实验操作也很简便，具 有广泛的底物适用性, 并以较高的收率得到预期产物.

2018 年，尚睿等 ${ }^{[34]}$ 报道了一种光催化 Heck 脱羧偶 联合成顺式烯烃化合物的方法(Scheme 21). 他们采用 将过渡金属催化剂与光氧化还原催化剂合并的策略，利 

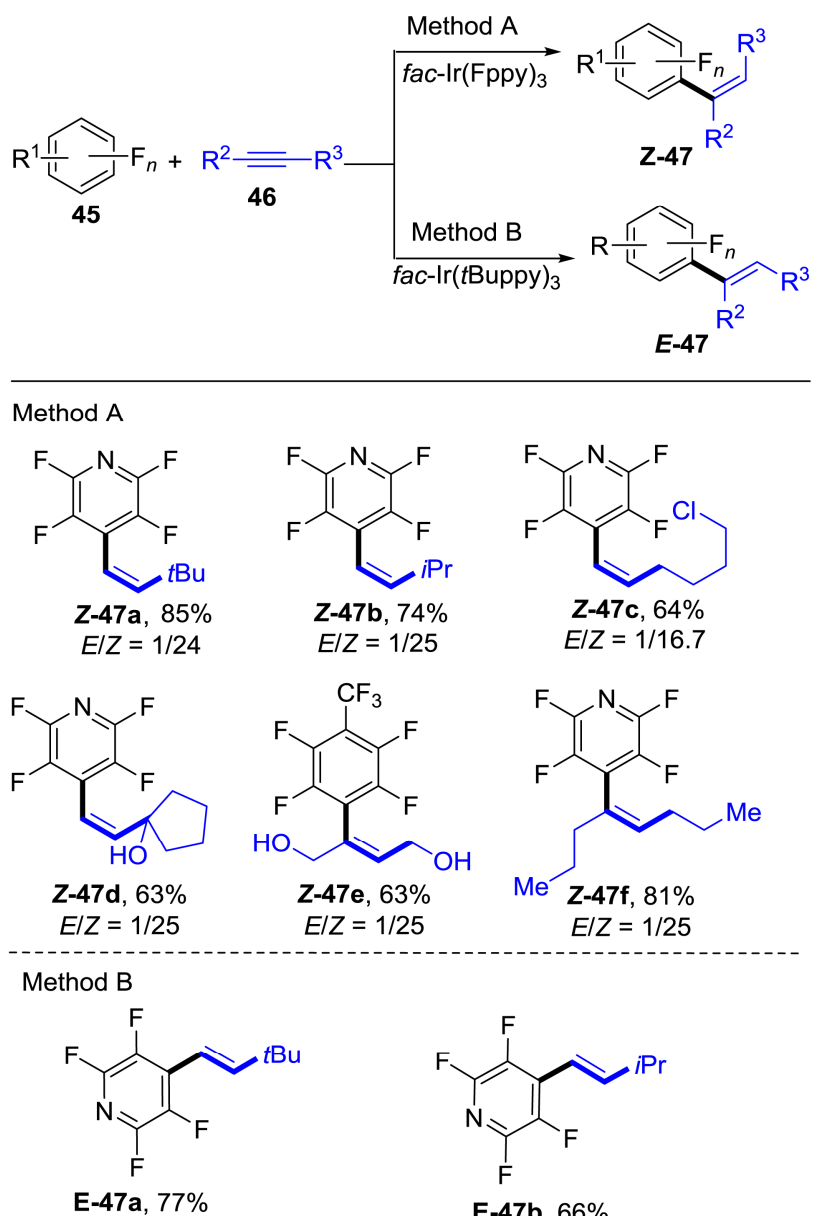

$-47 a, 77 \%$

$E / Z>25 / 1$
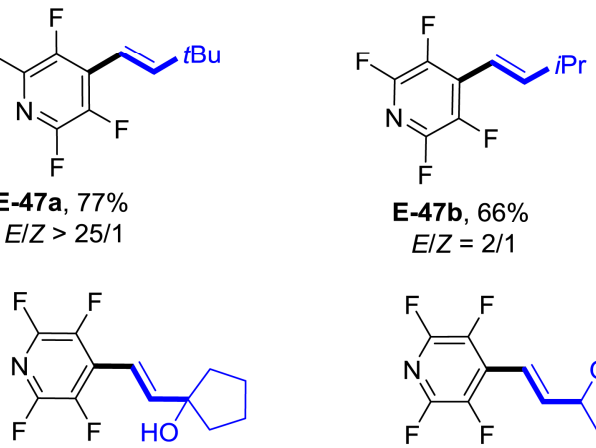

E-47c, $71 \%$

$E / Z=7 / 1$
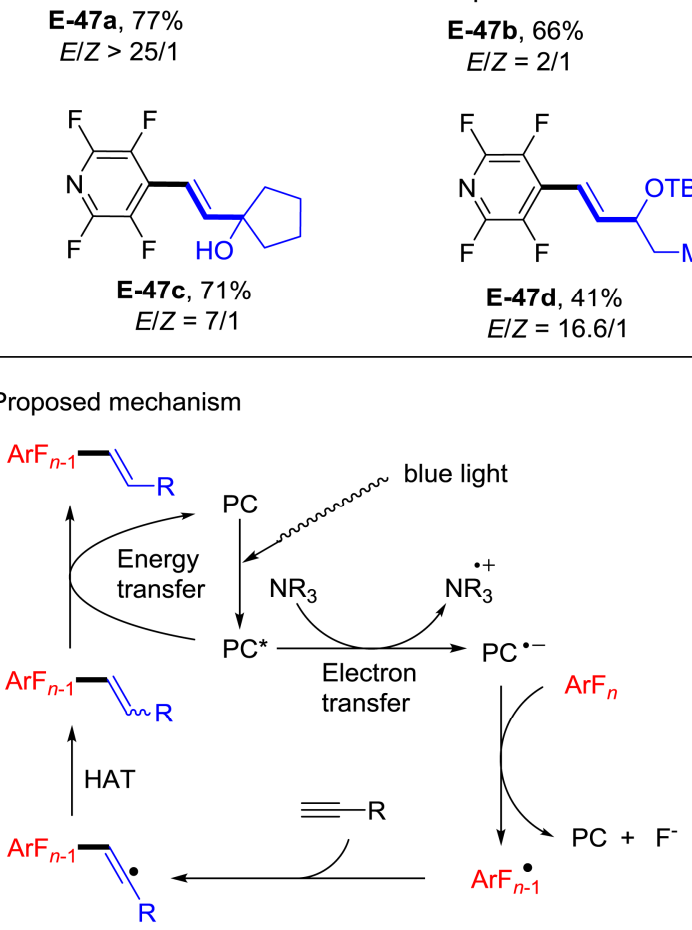

图式 18 光催化的 $\mathrm{C}-\mathrm{F}$ 键烯基化/异构化

Scheme 18 Photocatalytic $\mathrm{C}-\mathrm{F}$ alkenylation/isomerization

用生物质衍生物烷基羧酸 $\mathbf{5 8}$ 作为原料, 在 Heck 交叉偶 联反应中得到了良好 $Z$-选择性的取代苯乙烯衍生物 59.
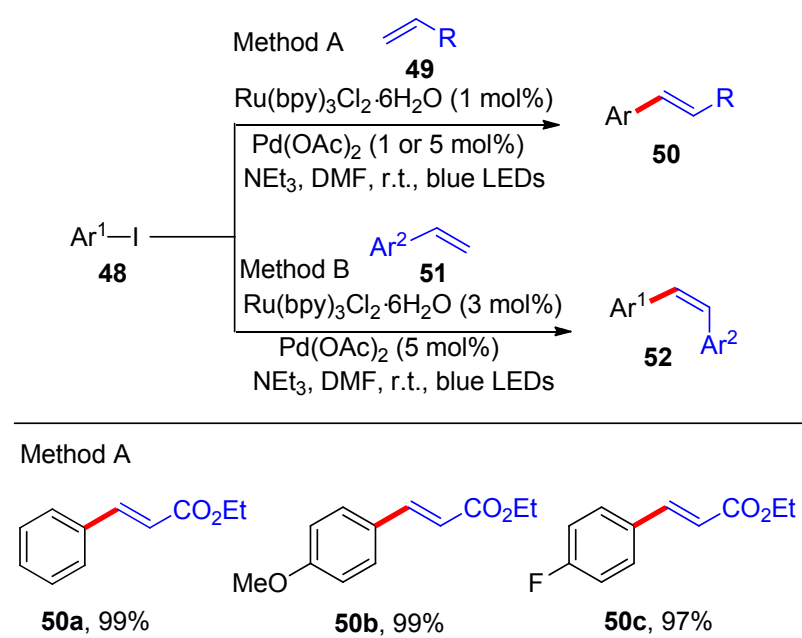<smiles>CCOC(=O)/C=C/c1ccccc1O[AsH3]</smiles><smiles>CCOC(=O)/C=C/c1ccccc1NC(C)(C)C</smiles>

$\mathbf{5 0 g}, 92 \% \quad 50 h, 93 \% \quad 50 i, 99 \%$<smiles>CCCCCCc1ccccc1</smiles>

52a, 95\% $Z / E=14 / 1$<smiles>Cc1cccc(/C=C\c2ccccc2)c1</smiles>

52d, 93\% ZIE $=8 / 1$<smiles>COc1ccc(/C=C\c2ccccc2)cc1</smiles>

52b, $89 \%$ $Z / E=9 / 1$<smiles>Cc1ccc(/C=C\c2ccc(C)cc2)cc1</smiles>

52e, $88 \%$

$Z / E=9 / 1$<smiles>Fc1ccc(/C=C\c2ccccc2)cc1</smiles>

52c, $93 \%$ ZIE $=21 / 1$
图式 19 可见光诱导的无配体的 Heck 反应 Scheme 19 Visible light-mediated Pd-Ru-catalyzed ligand-free Heck reactions

该反应展示了很好的烷基羧酸的底物范围，二级、三级 烷基羧酸以及 $\alpha$-氨基酸都可以很好的兼容 $(59 a \sim 590)$. 此外, 富电子和缺电子的取代苯乙烯也都有较好的反应 结果 $(59 p \sim 59 r)$. 这一新型的可见光促进的光催化烷基 羧酸脱羧偶联反应具有条件温和、绿色环保等优点, 为 脱翔偶联的发展提供了新的机遇.

程旭等 ${ }^{[35}$ 报道了一种取代苯乙烯的 1,5-溴三氯甲基 化反应，并对产物的构型进行了立体选择性控制 (Scheme 22). 通过量子产率实验和苂光淬灭实验, 他们 提出了一个可见光引发的串联反应的历程. $\alpha$-环丙烷苯 


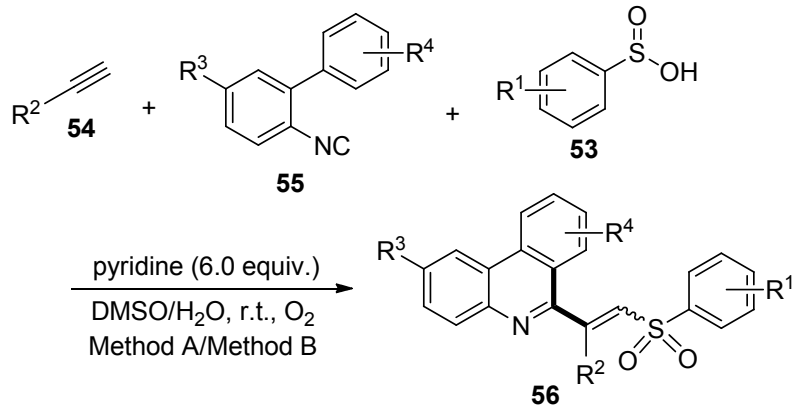

Method A: $3 \mathrm{~W}$ blue LED $(450 \pm 5 \mathrm{~nm})$ irradiation for $16 \mathrm{~h}$ Method B: 3 W UV ( $365 \pm 5 \mathrm{~nm})$ irradiation for $24 \mathrm{~h}$<smiles>CCCCCCC(=CS(=O)(=O)c1ccc(C)cc1)c1nc2ccccc2c2ccccc12</smiles>

Method A: E-56a

$71 \%, E / Z=10 / 1$

Method B: Z-56a

$73 \%, E / Z=1 / 16$

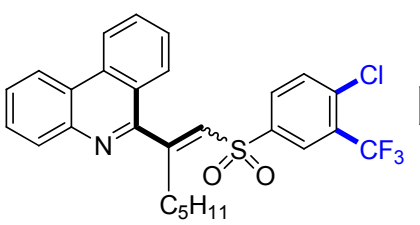

Method A: E-56c $60 \%, E / Z=9 / 1$

Method B: Z-56c $59 \%, E / Z=1 / 14$<smiles>CCCCCCCC(=Cc1nc2ccccc2c2ccc(Cl)cc12)S(=O)(=O)c1ccc(C)cc1</smiles>

Method A: E-56e

$63 \%, E / Z=11 / 1$

Method B: Z-56e

$68 \%, E / Z=1 / 13$

图式 20 光驱动的官能团化的菲啶类化合物的合成

Scheme 20 Photo-driven synthesis of functionalized phenanthridines

乙烯首先通过自由基链式反应机理进行 1,5-溴三氯甲基 化反应，生成的 $E$ 构型三取代苯乙烯类化合物进一步在 光照条件下发生构型翻转, 最终获得 $Z$ 构型产物, 产物 的 $Z / E$ 比例最高可达 99/1.

在此基础上，程旭等 ${ }^{[35]}$ 进一步探讨了三取代的反 式芳基烯丙醇 $\boldsymbol{E}-63$ 的 $E \rightarrow Z$ 异构化反应(Scheme 23). 研 究发现, 芳基取代基的电性以及 $\alpha$ 位的空间位阻对异构 化效率的影响不大, 都可以获得高收率和高选择性的 $Z$ 异构体.

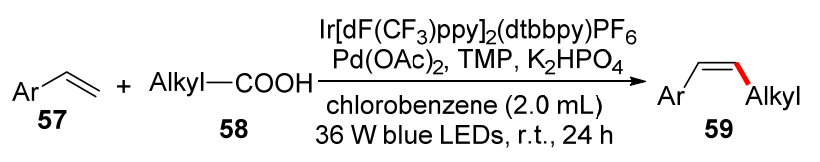

Tertiary aliphatic carto

59a, 58\%, ZIE $=99 / 1 \quad 59 b, 57 \%$, ZIE $=94 / 6$

$\mathrm{MeO}$
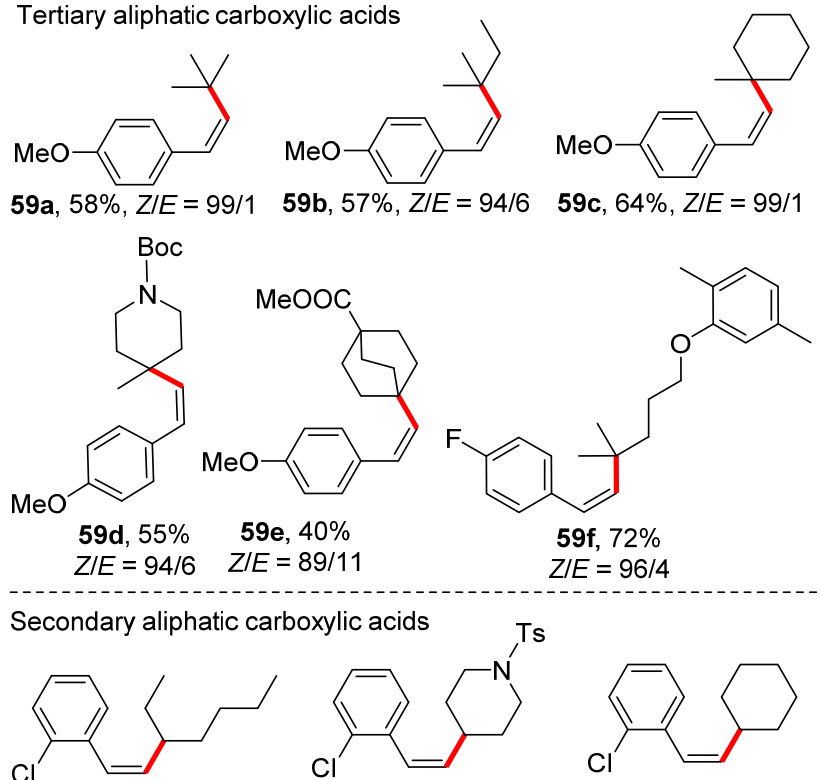

59g, 49\%, ZIE = 90/10 59h, 50\%, ZIE = 90/10 59i, 60\%, ZIE = 88/12 $\alpha$-amino acids

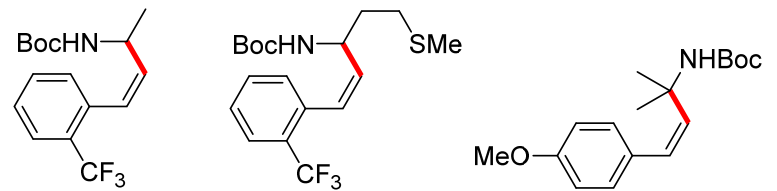

59j, $74 \%, Z I E=94 / 6 \quad 59 k, 33 \%, Z I E=95 / 5 \quad$ 59I, $60 \%, Z I E=94 / 6$

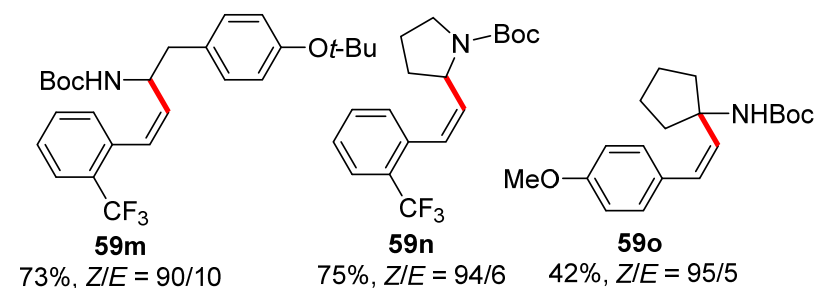

Scope with respect to vinyl arenes

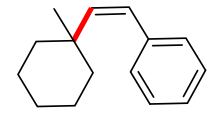

59 p, $60 \%$ $Z / E=92 / 8$

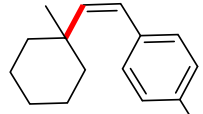

59q, 50\% $\quad \mathrm{CF}_{3}$ ZIE $=95 / 5$

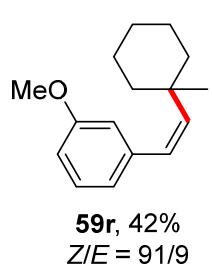

图式 21 光氧化还原/钯协同催化的 Z-选择性脱羧 Heck 反应 Scheme $21 Z$-Selective decarboxylative Heck reactions through photoredox/palladium catalysis

近期，俞寿云等 ${ }^{[36]}$ 报道了可见光催化的三组分参 与的自由基接力反应来制备 $\alpha$-氨甲基肉桂基醚 67 , 并 对其立体选择性进行了控制(Scheme 24). 发现当使用 DMSO 作为溶剂, 白色 LED 灯作为光源时, 可以快速地 得到 $E$ 构型为主的 $\alpha$-氨甲基肉桂基醚 $(\boldsymbol{E}-67)$, 产物的立 体选择性良好。该反应底物范围广泛，芳基乙烯基喼酸 
以及杂环乙烯基硼酸都可以很好地兼容. 此外, 富电子 和缺电子取代的芳基乙烯基硼酸也都有较好的反应结 果, 并且对各种官能团如酯、卤素、胺等皆有很好的容 忍度. 值得注意的是, 包含季碳中心的产物同样具有良 好的收率与选择性.

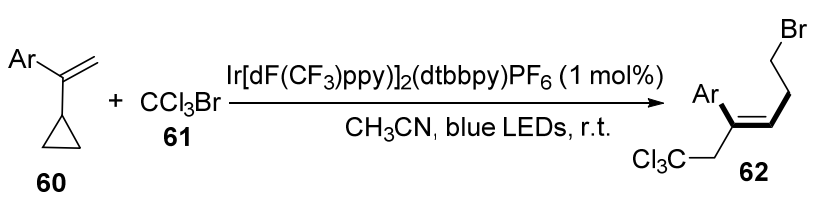

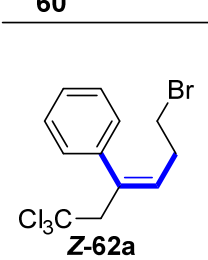

$91 \%, Z I E=96 / 4$

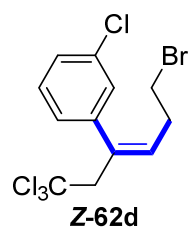

$89 \%, Z I E=96 / 4$

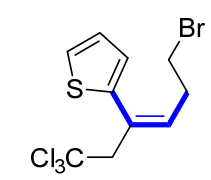

$\mathrm{Z}-62 \mathrm{~g}$

$70 \%, Z / E=75 / 25$

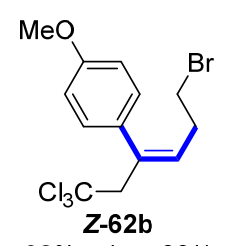

$92 \%, Z / E=99 / 1$

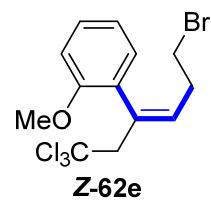

$81 \%, Z / E=83 / 17$

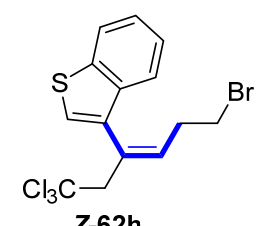

$90 \%, Z I E=99 / 1$

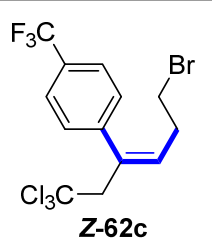

$89 \%$, ZIE $=92 / 8$

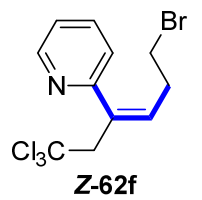

$92 \%, Z I E=99 / 1$

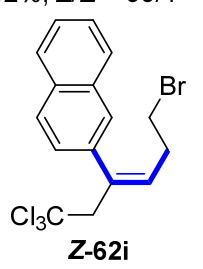

图式 22 1,5-溴三氯甲基化产物的 $E \rightarrow Z$ 异构化

Scheme $22 E \rightarrow Z$ isomerization of 1,5-bromotrichloromethylation reaction products

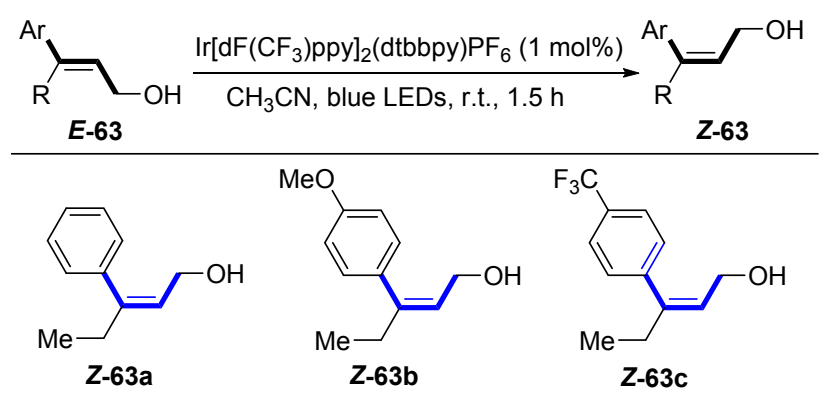

$94 \%, Z / E=99 / 1 \quad 95 \%, Z / E=96 / 4 \quad 96 \%, Z / E=99 / 1$

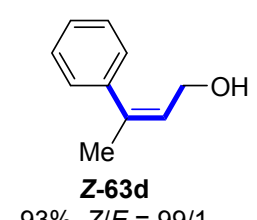

$93 \%, Z I E=99 / 1$

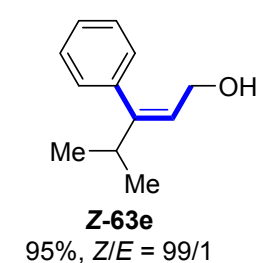

图式 23 三取代苯乙烯类化合物的 $E \rightarrow Z$ 异构化

Scheme $23 \quad E \rightarrow Z$ isomerization of trisubstituted styrenes

在此研究基础上, $(E)-\alpha$-氨甲基肉桂基醚 $(\boldsymbol{E}-67)$ 的 $E \rightarrow Z$ 异构化反应也被研究(Scheme 25). 研究发现, 光
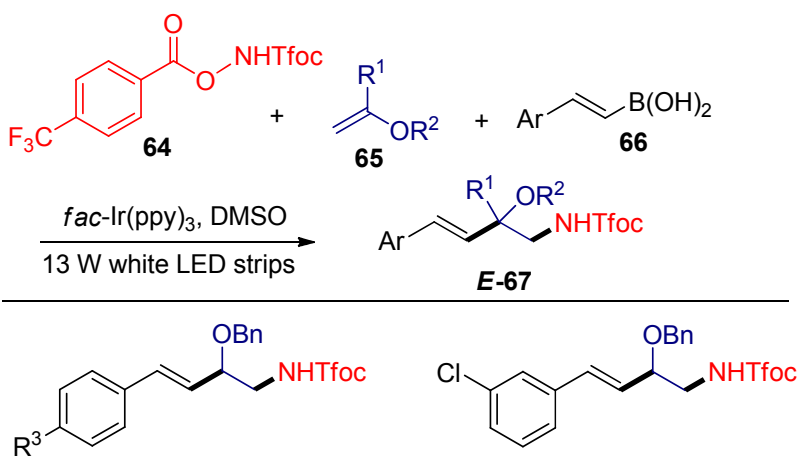

E-67a: $R^{3}=$ OMe, $64 \%, E / Z=92 / 8$

$E-67 b: R^{3}=\mathrm{CO}_{2} \mathrm{Me}, 60 \%, E / Z=75 / 25$

$E-67 \mathrm{c}: \mathrm{R}^{3}=\mathrm{Ph}, 64 \%, E / Z=81 / 19$

$E-67 d: R^{3}=t-B u, 90 \%, E / Z=90 / 10$

$E-67 \mathrm{e}: \mathrm{R}^{3}=\mathrm{F}, 87 \%, E / Z=90 / 10$

$E-67 f: R^{3}=C l, 67 \%, E / Z=83 / 17$

$E-67 \mathbf{g}, 91 \%, E / Z=86 / 14$

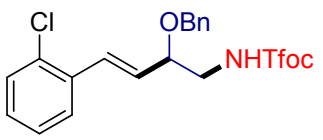

$E-67 \mathrm{~h}, 88 \%, E / Z=80 / 20$<smiles>CC(C)(C)OC(=O)NCC(C=Cc1cccc(NC(=O)c2ccccc2)c1)OCc1ccccc1</smiles>

$E-67 i, 79 \%, E / Z=86 / 14$<smiles>O=C(NCC(C=Cc1ccsc1)Oc1ccccc1)c1ccccc1</smiles>

$E-67 \mathbf{j}, 65 \%, E / Z=93 / 7$<smiles>O=C(NCC(C=Cc1cccc2ccccc12)OCc1ccccc1)c1ccccc1</smiles>

$E-67 \mathrm{k}, 66 \%, E / Z=86 / 14$<smiles>CCCCNCC(C=Cc1ccc2cc(OC)ccc2c1)OCCC</smiles>

$E-67 I, 61 \%, E / Z=81 / 19$

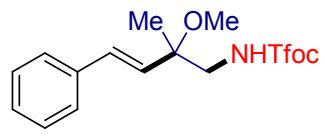

$E-67 \mathrm{~m}, 77 \%, E / Z>95 / 5$

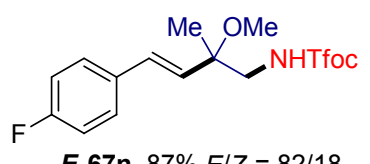

E-67n, $87 \% E / Z=82 / 18$

图式 24 自由基接力反应制备 $(E)$ - $\alpha$-氨甲基肉桂基醚 Scheme 24 Radical relay reaction for synthesizing $(E)$ - $\alpha$-aminomethyl cinnamyl ethers

源以及溶剂的选择对异构化反应至关重要. 经过条件篮 选，我们发现将第一步自由基接力反应得到的 $E$ 构型为 主的产物直接加入甲苯溶剂中, 在蓝色 LED 灯照射下, 即可实现 $E \rightarrow Z$ 异构化. 结果显示芳基乙烯基硼酸取 代基的电性以及空间位阻对异构化效率的影响都很小. 遗憾的是，包含季碳中心的产物顺式选择性并不理想.

\section{5 结论与展望}

对烯烃光化学异构化的策略以及促进光反应所需 的光催化剂类型进行分类并总结，同时对相应的反应机 理进行了讨论. 光催化烯烃的异构化反应，最早使用紫 外光为外加光源，但是由于紫外光能量较高，极易导致 副反应的发生，降低了反应收率，因此大大地限制了其 在有机合成中的应用. 近年来, 随着有机光化学的不断 发展，可见光的引入以及三线态光敏剂的不断开发，使 得可见光催化的策略成为一种新的有机合成的方法, 解 

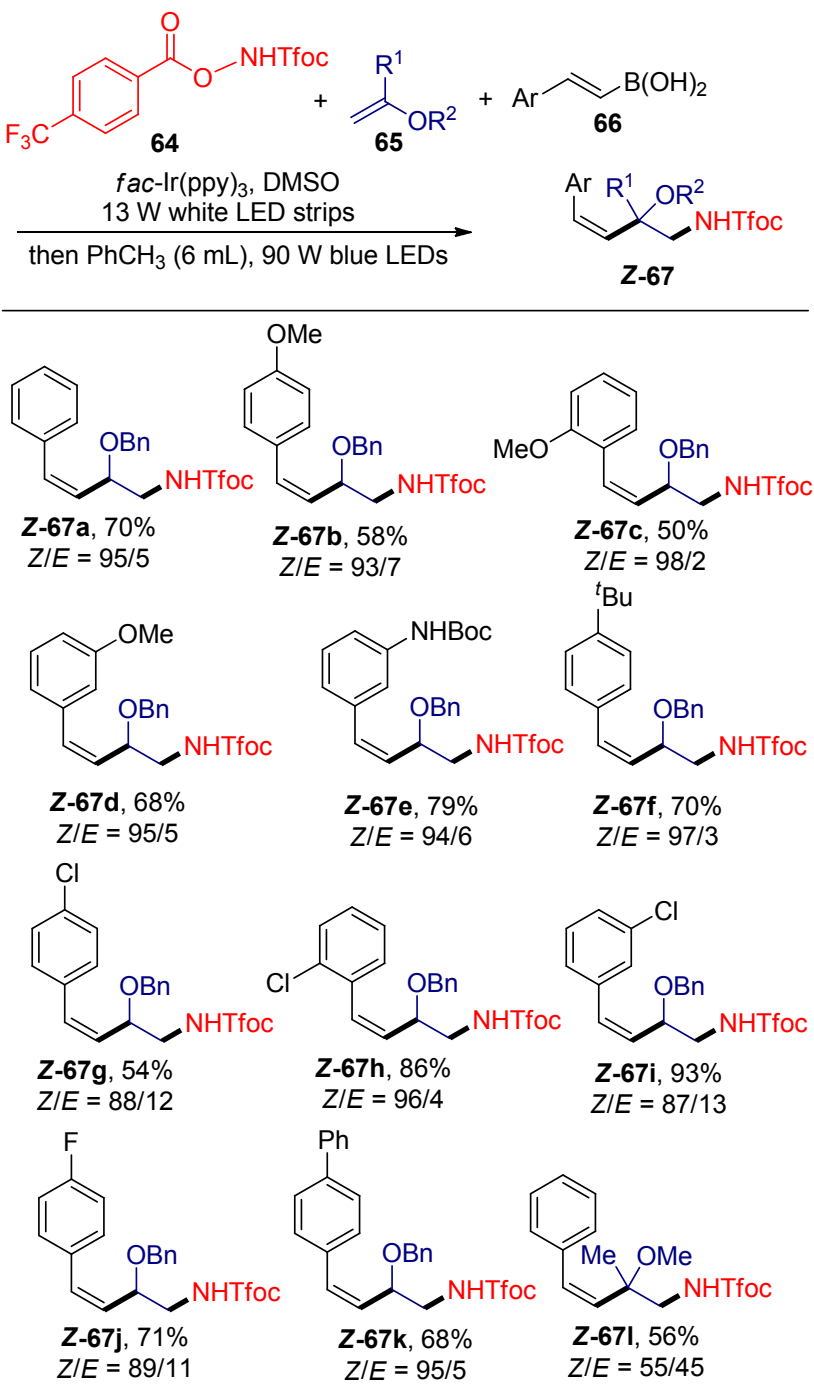

图式 25 自由基接力反应制备( $Z$ ) $-\alpha$-氨甲基肉桂基醚

Scheme 25 Radical relay reaction for synthesizing (Z)- $\alpha$-aminomethyl cinnamyl ethers

决了众多有机合成中的难点问题. 在烯烃的异构化, 尤 其是热力学不稳定的 $Z$-式烯烃的合成上取得了一系列 的突破. 反应操作简便, 条件温和, 大多数情况下室温 即可反应，原子经济性高.

可见光促进的烯烃异构化还存在一些问题, 需要克 服和解决. 首先, 绝大部分的烯烃底物都是苯乙烯的衍 生物, 非共轭的烯烃底物很难异构化; 其次, $E \rightarrow Z$ 异构 化研究较多, 反向异构化研究较少; 再次, 很多反应中, 异构化的选择性不够理想, 还需要进一步提高. 随着这 些问题的解决, 可以预见, 可见光促进的烯烃异构化反 应这一策略将成为立体选择性地构建烯烃的重要方法 之一.

\section{References}

[1] (a) Pearson, C. M.; Snaddon, T. N. ACS Cent. Sci. 2017, 3, 922. (b) Cai, W.; Fan, H.; Ding, D.; Zhang, Y.; Wang, W. Chem.
Commun. 2017, 53, 12918 .

(c) Singh, K.; Staig, S. J.; Weaver, J. D. J. Am. Chem. Soc. 2014, $136,5275$.

(d) Li, X.; Song, S.; Jiao, N. Acta Chim. Sinica 2017, 75, 1202 (in Chinese).

(李昕伟, 宋颂, 焦宁, 化学学报, 2017, 75, 1202.)

(e) Zhao, M.; Ji, Y. Chin. J. Org. Chem. 2018, 38, 401 (in Chinese). (赵明, 纪原, 有机化学, 2018, 38, 401.)

[2] (a) Wittig, G.; Geissler, G. Justus Liebigs Ann. Chem. 1953, 580, 44. (b) Bergelson, L. D.; Shemyakin, M. M. Tetrahedron 1963, 19, 149. (c) Dong, D. J.; Li, H.-H.; Tian, S.-K. J. Am. Chem. Soc. 2010, 132, 5018.

[3] (a) Julia, M.; Paris, J.-M. Tetrahedron Lett. 1973, 14, 4833.

(b) Yao, C.-Z.; Li, Q.-Q.; Wang, M.-M.; Ning, X.-S.; Kang, Y.-B. Chem. Commun. 2015, 51, 7729.

[4] (a) Peterson, D. J. J. Org. Chem. 1968, 33, 780.

(b) Staden, L. F. V.; Gravestock, D.; Ager, D. J. Chem. Soc. Rev. 2002, 31, 195 .

[5] (a) Koh, M. J.; Khan, R. K. M.; Torker, S.; Hoveyda, A. H. Angew. Chem., Int. Ed. 2014, 53, 1968.

(b) Quigley, B. L.; Grubbs, R. H. Chem. Sci. 2013, 5, 501.

(c) Gottumukkala, A. L.; Madduri, A. V. R.; Minnaard, A. J. ChemCatChem 2012, 4, 462.

(d) Meek, S. J.; O'Brien, R. V.; Llaveria, J.; Schrock, R. R.; Hoveyda, A. H. Nature 2011, 471, 461

(e) Endo, K.; Grubbs, R. H. J. Am. Chem. Soc. 2011, 133, 8525.

[6] (a) Lindlar, H. Helv. Chim. Acta 1952, 35, 446.

(b) Li, X.; Song, W.; Tang, W. J. Am. Chem. Soc. 2013, 135, 16797. (c) Song, W.; Li, X.; Yang, K.; Zhao, X.-L.; Glazier, D. A.; Xi, B.-M.; Tang, W. J. Org. Chem. 2016, 81, 2930.

[7] Singh, K.; Staig, S. J.; Weaver, J. D. J. Am. Chem. Soc. 2014, 136, 5275.

[8] Cai, W.; Fan, H.; Ding, D.; Zhang, Y.; Wang, W. Chem. Commun. 2017, 53, 12918

[9] (a) Wald, G. J. Gen. Physiol. 1935, 19, 351.

(b) O’Leary, B.; Duke, B.; Eilers, J. E. Nature 1973, 246, 166.

(c) Lion, F.; Rotmans, J. P.; Daemen, F. J. M.; Bonting, S. L. Biochim. Biophys. Acta 1975, 384, 283.

(d) Gai, F.; Hasson, K. C.; Cooper McDonald, J.; Anfinrud, P. A Science 1998, 279, 1886.

(e) Rando, R. R. Chem. Rev. 2001, 101, 1881

(f) Strauss, O. Physiol. Rev. 2005, 85, 845.

(g) Redmond, T. M.; Poliakov, E.; Yu, S.; Tsai, J.-Y.; Lu, Z.; Gentleman, S. Proc. Natl. Acad. Sci. U. S. A. 2005, 102, 13658.

[10] Metternich, J. B.; Gilmour, R. Synlett 2016, 27, 2541.

[11] (a) Metternich, J. B.; Gilmour, R. J. Am. Chem. Soc. 2015, 137, 11254 .

(b) Metternich, J. B.; Artiukhin, D. G.; Holland, M. C.; Bremen-Kühne, M. V.; Neugebauer, J.; Gilmour, R. J. Org. Chem. 2017, 82, 9955.

[12] Walker, A. G.; Radda, G. K. Nature 1967, 215, 1483.

[13] Metternich, J. B.; Gilmour, R. J. Am. Chem. Soc. 2015, 137, 11254.

[14] Metternich, J. B.; Gilmour, R. J. Am. Chem. Soc. 2016, 138, 1040.

[15] Metternich, J. B.; Artiukhin, D. G.; Holland, M. C.; Bremen-Kühne, M. V.; Neugebauer, J.; Gilmour, R. J. Org. Chem. 2017, 82, 9955

[16] Metternich, J. B.; Sagebiel, S.; Lückener, A.; Lamping, S.; Ravoo, B. J.; Gilmour, R. Chem.-Eur. J. 2018, 24, 4228.

[17] Hammond, G. S.; Saltiel, J.; Lamola, A. A.; Turro, N. J.; Bradshaw, J. S.; Cowan, D. O.; Counsell, R. C.; Vogt, V.; Dalton, C. J. Am. Chem. Soc. 1964, 86, 3197 .

[18] (a) Hammond, G. S.; Saltiel, J. J. Am. Chem. Soc. 1962, 84, 4983. (b) Hammond, G. S.; Saltiel, J. J. Am. Chem. Soc. 1963, 85, 2515. (c) Hammond, G. S.; Saltiel, J.; Lamola, A. A.; Turro, N. J.; Bradshaw, J. S.; Cowan, D. O.; Counsell, R. C.; Vogt, V.; Dalton, C. J. Am. Chem. Soc. 1964, 86, 3197.

[19] (a) Arai, T.; Sakuragi, H.; Tokumaru, K. Chem. Lett. 1980, 9, 261. (b) Arai, T.; Sakuragi, H.; Tokumaru, K. Bull. Chem. Soc. Jpn. 1982, 55, 2204. 
[20] Zhao, Y.-P.; Yang, L.-Y.; Liu, R. S. H. Green Chem. 2009, 11, 837.

[21] Cai, W.; Fan, H.; Ding, D.; Zhang, Y.; Wang, W. Chem. Commun. 2017, 53, 12918.

[22] Faßbender, S. I.; Metternich, J. B.; Gilmour, R. Org. Lett. 2018, 20, 724.

[23] Osawa, M.; Hoshino, M.; Wakatsuki, Y. Angew. Chem., Int. Ed. 2001, 40,18

[24] Rackl, D.; Kreitmeier, P.; Reiser, O. Green Chem. 2016, 18, 214.

[25] Fabry, D. C.; Ronge, M. A.; Rueping, M. Chem. Eur. J. 2015, 21, 5350.

[26] Molloy, J. J.; Metternich, J. B.; Daniliuc, C. G.; Watson, A. J. B.; Gilmour, R. Angew. Chem., Int. Ed. 2018, 57, 3168.

[27] Lin, Q.-Y.; Xu, X.-H.; Qing, F.-L. J. Org. Chem. 2014, 79, 10434.

[28] Iqbal, N.; Jung, J.; Park, S.; Cho, E. J. Angew. Chem., Int. Ed. 2014, 53,539 .
[29] Senaweera, S. M.; Singh, A.; Weaver, J. D. J. Am. Chem. Soc. 2014, 136, 3002

[30] (a) Singh, A.; Kubik, J. J.; Weaver, J. D. Chem. Sci. 2015, 6, 7206. (b) Senaweera, S.; Weaver, J. D. J. Am. Chem. Soc. 2016, 138 , 2520.

[31] Singh, A.; Fennell, C. J.; Weaver, J. D. Chem. Sci. 2016, 7, 6796.

[32] Zhang, H.; Huang, X. Adv. Synth. Catal. 2016, 358, 3736.

[33] Li, Y.; Miao, T.; Li, P.; Wang, L. Org. Lett. 2018, 20, 1735.

[34] Zheng, C.; Cheng, W.-M.; Li, H.-L.; Na, R.-S.; Shang, R. Org. Lett. 2018, 20, 2559.

[35] Li, J.; Chen, J.; Huang, W.; Cheng, X. Chin. J. Org. Chem. 2018, $38,1507$.

(李进, 陈靖之, 黄文浩, 程旭, 有机化学, 2018, 38, 1507.)

[36] An, X.-D.; Zhang, H.; Xu, Q.; Yu, L.; Yu, S. Chin. J. Chem. 2018, $36,1147$.

(Lu, Y.) 